\title{
Iterated greedy local search methods for unrelated parallel machine scheduling
}

\author{
Luís Fanjul Peyró, Rubén Ruiz* \\ Grupo de Sistemas de Optimización Aplicada, Instituto Tecnológico de Informática, \\ Universidad Politécnica de Valencia, Valencia, Spain. \\ lfpeyro@terra.es, rruiz@eio.upv.es
}

July 15, 2009

\begin{abstract}
This work deals with the parallel machines scheduling problem which consists in the assignment of $n$ jobs on $m$ parallel machines. The most general variant of this problem is when the processing time depends on the machine to which each job is assigned to. This case is known as the unrelated parallel machines problem. Similarly to most of the literature, this paper deals with the minimization of the maximum completion time of the jobs, commonly referred to as makespan $\left(C_{\max }\right)$. Many algorithms and methods have been proposed for this hard combinatorial problem, including several highly sophisticated procedures. By contrast, in this paper we propose a set of simple iterated greedy local search based metaheuristics that produce solutions of very good quality in a very short amount of time. Extensive computational campaigns show that these solutions are, most of the time, better than the current state-of-the-art methodologies by a statistically significant margin.
\end{abstract}

Keywords: unrelated parallel machines, makespan, iterated greedy, local search

\section{Introduction}

The parallel machine scheduling problem is a typical shop configuration where there is a set $N$ of $n$ independent jobs that have to be processed on a set $M$ of $m$ machines disposed in parallel. Each

\footnotetext{
*Corresponding author. Tel: +34 9638770 07, ext: 74946. Fax: +34 963877499
} 
job $j, j=1, \ldots, n$ has to be processed by exactly one out of the $m$ parallel machines. No machine can process more than one job at the same time. Furthermore, once the processing of a job by a given machine has started, it has to continue until completion. The processing time of a job is a known, finite and fixed positive number referred to as $p_{j}$, i.e., any of the $m$ machines will be occupied by $p_{j}$ units of time when processing job $j$. This is known as the identical parallel machine scheduling problem case as each job has the same processing time requirements, regardless of the machine employed. The uniform parallel machine case arises when each machine $i, i=1, \ldots, m$ has a different speed $s_{i}$ for processing all the jobs. Therefore, the processing time of a job $j$ on machine $i$ is derived as follows: $p_{i j}=p_{j} / s_{i}$. The most general setting comes when the processing time of each job depends on the machine where it is processed. This last scenario is referred to as the unrelated parallel machines scheduling problem. The input data for this problem is $n, m$ and a matrix with the processing times $p_{i j}$. One of the most commonly studied objectives in parallel machine scheduling problems is the maximum completion time (or $C_{\max }$ ) minimization. According to the well known $\alpha / \beta / \gamma$ scheduling problems classification scheme proposed initially by Graham et al. (1979), the problem dealt with in this paper is denoted as $R / / C_{\max }$.

The $R / / C_{\max }$ problem as considered above is, in reality, an assignment problem. This is because the processing order of the jobs assigned to a given machine do not alter the maximum completion time at that machine. Therefore, there are $m^{n}$ possible solutions to the problem after all possible assignments. The $R / / C_{\max }$ has been shown to be $\mathcal{N} \mathcal{P}$-Hard in the strong sense, since the special case with identical machines (referred to as $P / / C_{\max }$ ) was already demonstrated by Garev and Johnson (1979) to belong to that class. Even the two machine version $\left(P 2 / / C_{\max }\right)$ is already $\mathcal{N} \mathcal{P}$-Hard according to Lenstra et al. (1977). Furthermore, Lenstra et al. (1990) showed that no polynomial time algorithm exists for the general $R / / C_{\max }$ problem with a better worstcase ratio approximation than $3 / 2$ unless $\mathcal{P}=\mathcal{N} \mathcal{P}$. A Mixed Integer Linear Programming (MILP) formulation for the $R / / C_{\max }$ is provided for the sake of completeness. Let $x_{i j}$ be the binary assignment variable, which is equal to 1 (respectively 0) if job $j$ is assigned (respectively not assigned) to machine $i$. The MILP model is then:

$$
\begin{gathered}
\min C_{\max } \\
\sum_{i=1}^{m} x_{i j}=1 \quad \forall j \in N \\
\sum_{j=1}^{n} p_{i j} \cdot x_{i j} \leq C_{\max } \quad \forall i \in M \\
x_{i j} \in\{0,1\} \quad \forall j \in N, \forall i \in M
\end{gathered}
$$

It is common to find applications that can be modeled by an instance of $R / / C_{\max }$. For 
example, on mass production lines there is usually more than one machine that can carry out the production tasks. Other examples are: docking systems for ships, multiprocessor computers, and many others. Some additional examples can be obtained from Pinedo (2005), Pinedo (2008) or Sule (2008).

As we will show, the existing literature on the $R / / C_{\max }$ problem already contains highly effective methods. However, many state-of-the-art algorithms either need commercial solvers which might not be available in all situations or are somewhat intricate. The research question in this paper is if similar top performance can be obtained with more general and simpler heuristics. More specifically, in this work we propose new metaheuristics based on the application of the recently introduced Iterated Greedy (IG) methodology for scheduling problems (Ruiz and Stützle, 2007). As we will detail, IG methods coupled with fast local search with different neighborhoods, based on the Variable Neighborhood Descent approach (VND, Mladenovic and Hansen, 1997; Hansen and Mladenovic, 2001) results in a simpler approach to the $R / / C_{\max }$ without sacrificing state-of-the-art results.

The organization of this paper is as follows: In Section 2, some of the classical as well as the recent literature is reviewed. Section 3 details the algorithms proposed. Extensive computational and statistical analyses are presented in Section 4. Finally, some concluding remarks and future research directions are given in Section 5 ,

\section{Literature review}

Parallel machine scheduling was already studied in the late fifties with the work of McNaughton (1959). Later, in Graham (1969), dispatching rules were proposed for the identical parallel machines case and no precedence constraints among jobs. More specifically, the author studied the application of the Longest Processing Time first (LPT) dispatching rule that guaranteed a worst case error bound of $2-1 / \varepsilon$. The literature on parallel machine scheduling is fairly large, for an in-depth review, we refer the readers to the reviews of Cheng and Sin (1990) and to the more recent one by Mokotoff (2001). In what follows, and due to reasons of space, we focus mainly on the non-preemptive unrelated parallel machine problem with makespan criterion.

Horowitz and Sahni (1976) proposed a Dynamic Programming exact approach and some approximated methods for the $R 2 / / C_{\max }$ problem (and also for flowtime criterion). Ibarra and Kim (1977) presented five approximation methods for the two machine and $m$-machines cases together with proof that the LPT rule has a very tight error bound for $C_{\max }$ and large values of $m$. De and Morton (1980) proposed heuristics with very good performance for relatively small problems. Davis and Jaffe (1981) proposed an approximation algorithm with a worst case error bound of $2 \sqrt{m}$. Later, Lenstra et al. (1990) showed another heuristic with a better worst case ratio of 2 .

A large body of research efforts have been concentrated on the idea of solving the linear relaxation of the MILP model presented in Section 1 by dropping the integrality constraints in 
(44) and taking into account constraints (5) below instead.

$$
x_{i j} \geq 0 \quad \forall j \in N, \forall i \in M
$$

The optimum linear solution to the relaxed MILP is obtained in a first phase. In a second phase, a rounding method is applied in order to obtain a feasible solution to the problem. This rounding method or rounding phase can be either exact or approximated. Potts (1985) was the first to employ this technique, which was later exploited and refined, among others, by Lenstra et al. (1990), Shmoys and Tardos (1993) and more recently, by Shchepin and Vakhania (2005). In this last paper, the authors improved the earlier best known error bound of Lenstra et al. (1990) from 2 to $2-1 / m$. Note that this "two-phase approach" often requires of an efficient linear programming solver for the first phase.

Metaheuristics have provided very good results for the $R / / C_{\max }$. Hariri and Potts (1991) proposed some heuristics complemented with local search improvement methods and showed promising results. In 1993, van de Velde proposed two algorithms, an exact one and an iterated local search metaheuristic, both of them based on the surrogate relaxation and duality of the MILP model presented before. Problems of up to 200 jobs and 20 machines $(200 \times 20)$ were tested. The exact method was able to solve instances up to $200 \times 2$ or up to $50 \times 20$. The local search procedure had relatively large deviations from the optimum solutions in most problems, specially in those with larger $m$ values. To the best of our knowledge, Glass et al. (1994) were the first in proposing a Genetic Algorithm (GA), Tabu Search (TS) and Simulated Annealing (SA) algorithms. Under some conditions, all three algorithms (standard versions) provided comparable results, although for larger computation times, the proposed GA and SA showed better performance. Later, Piersma and van Diik (1996) presented a SA and a TS with initializations coming from the heuristics of Hariri and Potts (1991) and Davis and Jaffe (1981). The proposed algorithms included an effective local search with very good results at the time.

In the literature we also find some exact approaches with excellent results. Martello et al. (1997) proposed a Branch and Bound (B\&B) method using effective lower bounds and some heuristics for the upper bounds. The results exceeded those of van de Velde (1993) providing relatively small errors for instances of up to $80 \times 20$. Despite this good results, research has continued in metaheuristics. For example, Srivastava (1998) proposed an advanced TS. Later, Sourd (2001) presented two methods based on large neighborhood search. The first does a heuristic partial tree exploration and the second one is also based on the duality of the MILP model employed by van de Velde (1993).

An interesting proposal was put forward by Mokotoff and Chretienne (2002). The authors developed a cutting planes method which basically selects first a subset of the constraints of the previous MILP model. An optimal solution to this simplified model is obtained. The original constraints are checked. If all of them are satisfied, the optimal solution to the original problem is given. If some of the original constraints are violated, then one or more constraints are 
added to the MILP simplification and the model is solved again. Notice how this methodology is different that the previously commented two-phase approach. This technique was later refined in Mokotoff and Jimeno (2002). In that paper, an algorithm dubbed as "Partial" is presented. Partial is based on the methodology of Dillenberger et al. (1994) which was later used by Mansini and Speranza (1999). In general, Partial is based on solving the previous MILP model with less binary variables (notice the difference with the paper of Mokotoff and Chretienne (2002) where instead of less variables, less constraints are tested). As a result, the optimal solution of the reduced MILP might have some non-integer variables for the assignment of jobs to machines. These are rounded in a second phase in the search for good solutions. This novel methodology provided excellent results, solving instances of up to $200 \times 20$ in short CPU times of less than 75 seconds with small relative percentage deviations from optimum solutions of less than $2 \%$. Both methodologies proposed in Mokotoff and Chretienne (2002) and in Mokotoff and Jimeno (2002) make extensive use of commercial solvers for their respective initial phases.

In an excellent work, Woclaw (2006) carried out a comprehensive re-implementation and a careful computational evaluation of most (if not all) existing literature for the $R / / C_{\max }$. The author tested and evaluated many heuristics, metaheuristics and exact methods in a comparable scenario and with the same instance benchmark. From the results, the Partial method of Mokotoff and Jimeno (2002) was concluded to be state-of-the-art.

After the publication of Woclaw' thesis, Ghirardi and Potts (2005) published a work showing excellent results. A Recovering Beam Search (RBS) approach is proposed. The RBS methodology was already studied by de la Croce et al. (2004), which is in turn based on the known Beam Search (BS) method of Ow and Morton (1988), among others. BS basically truncates the B\&B allowing the exploration of only the $w$ most promising nodes at each level of the search tree. $w$ is the beam width. RBS is an improvement of BS in which there is a recovery phase where a given solution $s$ is checked to see if it is dominated by another partial solution $s_{0}$ in the same level of the tree. If this is the case, $s$ is discarded and $s_{0}$ replaces $s$ as the new incumbent partial solution. Instances of up to $1000 \times 50$, were tested, with good results under 150 seconds CPU time.

Some other recent algorithms have appeared. For example, Gairing et al. (2007) revisits the two-phase approach. The authors propose a very fast method albeit with results that cannot compete with those of Mokotoff and Jimeno (2002) or Ghirardi and Potts (2005). From a close observation of the recent literature, it seems that the Partial and RBS methods proposed in these last two papers, respectively, are the current state-of-the-art.

We would like to finish the literature review with an observation. To the best of our knowledge, no author compares the results of the different proposed strategies for solving the $R / / C_{\max }$ problem against a modern and effective solver. As we will later show, the simplistic MILP model shown in Section 1 is solved quite effectively with a standard commercial solver like for example IBM-ILOG CPLEX 11.0. which is based in current Branch \& Cut methodologies. 


\section{Proposed methods}

In this paper, we are mainly interested in simple local search based methods for the $R / / C_{\max }$ problem. In what follows, we detail the different proposals which range from simpler to more sophisticated but always retaining a conceptual simplicity. Before entering into details, we define some additional notation. Let us refer to $J_{i}$ as to the set of jobs that have been assigned to machine $i, i \in M$. $C_{i}$ is the time at which machine $i$ finishes processing of its $J_{i}$ assigned jobs, i.e., $C_{i}=\sum_{j \in J_{i}} p_{i j}$.

All proposed methods share a common structure. First an initial solution is obtained with a very naïve heuristic. Then we enter in a loop that is repeated until a given termination criterion is satisfied which in this paper is a given elapsed CPU time. In this loop we apply two consecutive simple local search methods based on two different neighborhoods until the solution is a local optimum with respect both neighborhood definitions. This is the Variable Neighborhood Descent (VND) loop (Hansen and Mladenovic, 2001). After the VND loop, a given algorithm is applied to "modify" the solution. This modification is either based on an Iterated Greedy (IG) method (Ruiz and Stützle, 2007) or on a Restricted Local Search (RLS) procedure. After the modification, the VND loop is applied again. This common structure or template is detailed in Figure 1. In the following sections we detail all the elements of the proposed methods and further elaborate on the previous template.

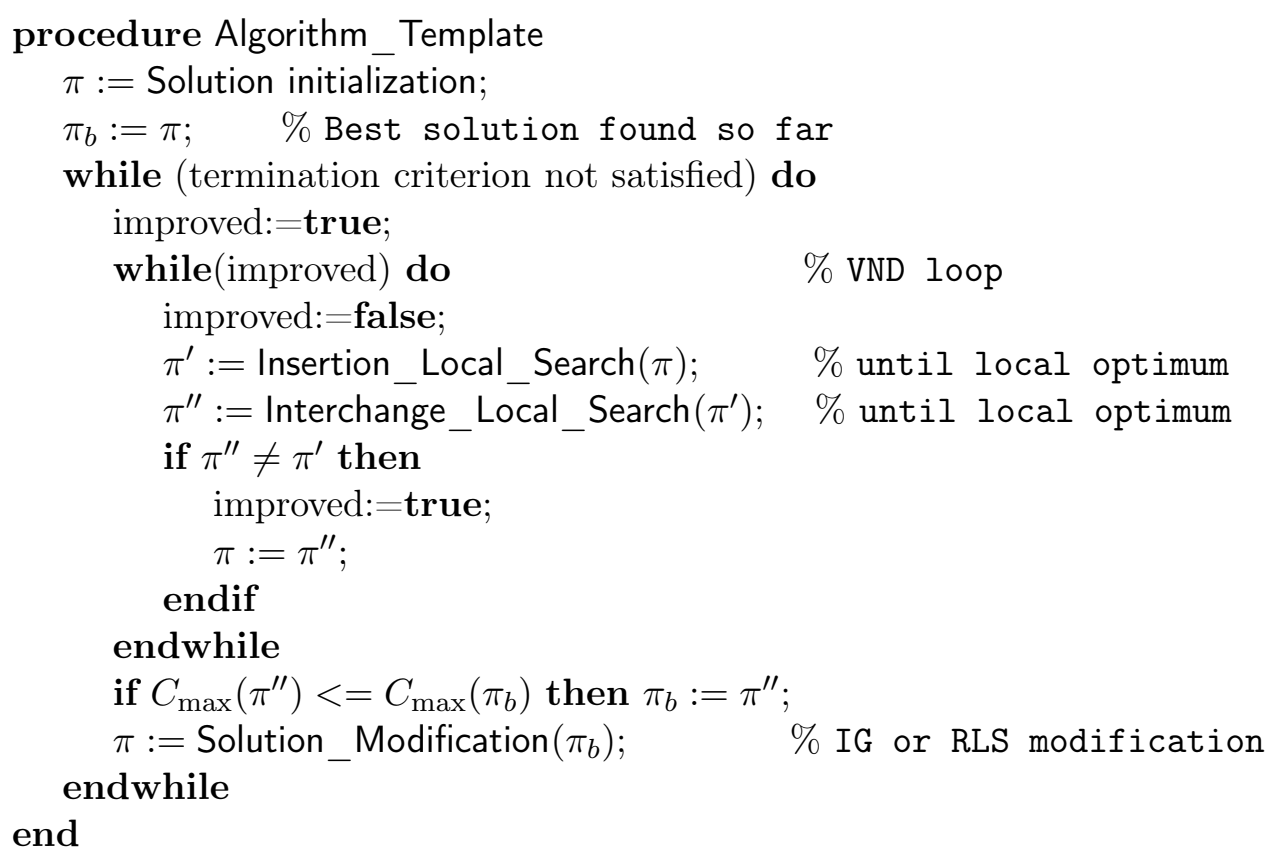

Figure 1: Pseudo algorithm template for all proposed methods. 


\subsection{Solution initialization and local search procedures}

We are interested in a fast and simple initial solution. Basically, we assign each job to the fastest machine, i.e., we assign each job $j$ to the machine $l=\underset{i \in M}{\operatorname{argmin}}\left\{p_{i j}\right\}$. This is an extremely fast initialization with a computational cost of $\mathcal{O}(\mathrm{nm})$ steps. As we will comment later on, there is no need for more sophisticated initializations.

The VND loop iteratively applies Insertion and Interchange local searches, each one of them until a local optimum is reached.

We define the insertion neighborhood for the $R / / C_{\max }$ problem as all sequences where one job is extracted from one machine and assigned to another machine. The cardinality of this neighborhood is $n(m-1)$ and it is very easy to evaluate since each movement requires a substraction and an addition. We have a job $j$ assigned to a machine $i$. We evaluate the lowest completion time when assigning job $j$ to all other machines $h \in M / i$. We call $l$ to the machine with the lowest completion time after assigning job $j$, i.e., $l=\underset{h \in M / i}{\operatorname{argmin}}\left\{C_{h}+p_{h j}\right\}$. The movement is accepted if $C_{l}+p_{l j}<C_{i}$ (which in turn ensures that $C_{l}+p_{l j}<C_{\max }$ ). One single pass of this insertion local search has a computational complexity of $\mathcal{O}(n m)$. Figure 2 shows this procedure in more detail.

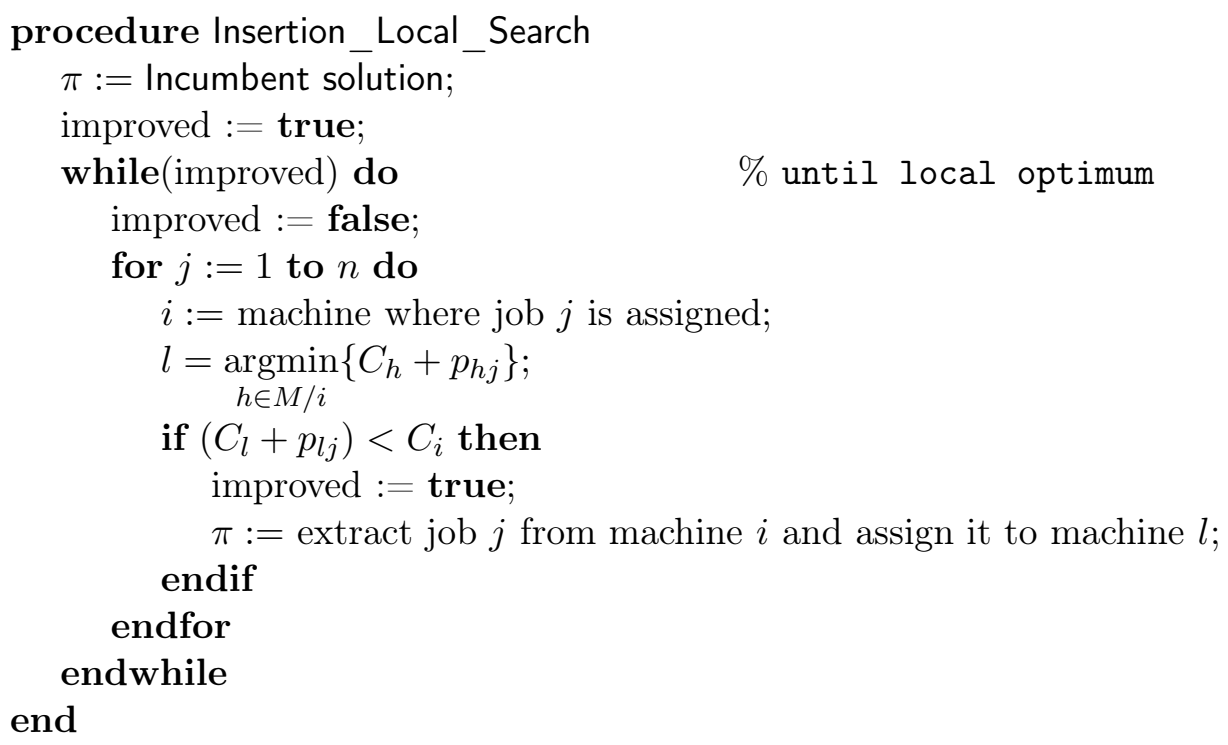

Figure 2: Insertion Local Search procedure employed in the VND phase.

The second neighborhood has a larger cardinality and involves two jobs $j_{1}$ and $j_{2}$ assigned to two different machines $i_{1}$ and $i_{2}$. The movement consists in extracting job $j_{1}\left(j_{2}\right)$ from machine $i_{1}\left(i_{2}\right)$ and assigning it to machine $i_{2}\left(i_{1}\right)$. Obviously, in order for the movement to be accepted, the completion times of $i_{1}$ and $i_{2}$ after the interchange should be lower than the $C_{\max }$. Furthermore, 
we seek a net gain in the processing times, i.e., $p_{i_{1} j_{2}}+p_{i_{2} j_{1}}$ must be lower than $p_{i_{1} j_{1}}+p_{i_{2} j_{2}}$, otherwise, the change, albeit not deteriorating the $C_{\max }$ value, increases the net completion times of $i_{1}$ and $i_{2}$ and is not, in most cases, beneficial. The cardinality of this neighborhood is harder to calculate since no interchange movements are carried out among jobs assigned to the same machine but in the worst case it is $n(n-1)$ which amounts to a computational complexity of $\mathcal{O}\left(n^{2}\right)$. Figure 3 depicts the whole procedure in a pseudo-algorithm form. Notice that this neighborhood is much larger than the insertion one and one possible speed up is to use the first improvement strategy for each job. In other words, the local search moves to the next job as soon as a movement is accepted for the current job. Notice also that Figure 3 shows a clear description of the interchange local search, not the best possible implementation. The implementation we have used takes job $j_{1}$ and interchanges it with all jobs in all machines except $i_{1}$. In this way we save checking if $j_{1} \neq j_{2}$ and if $i_{1} \neq i_{2}$. However, all pseudo-algorithm listings in this paper have been constructed for clarity, not efficiency.

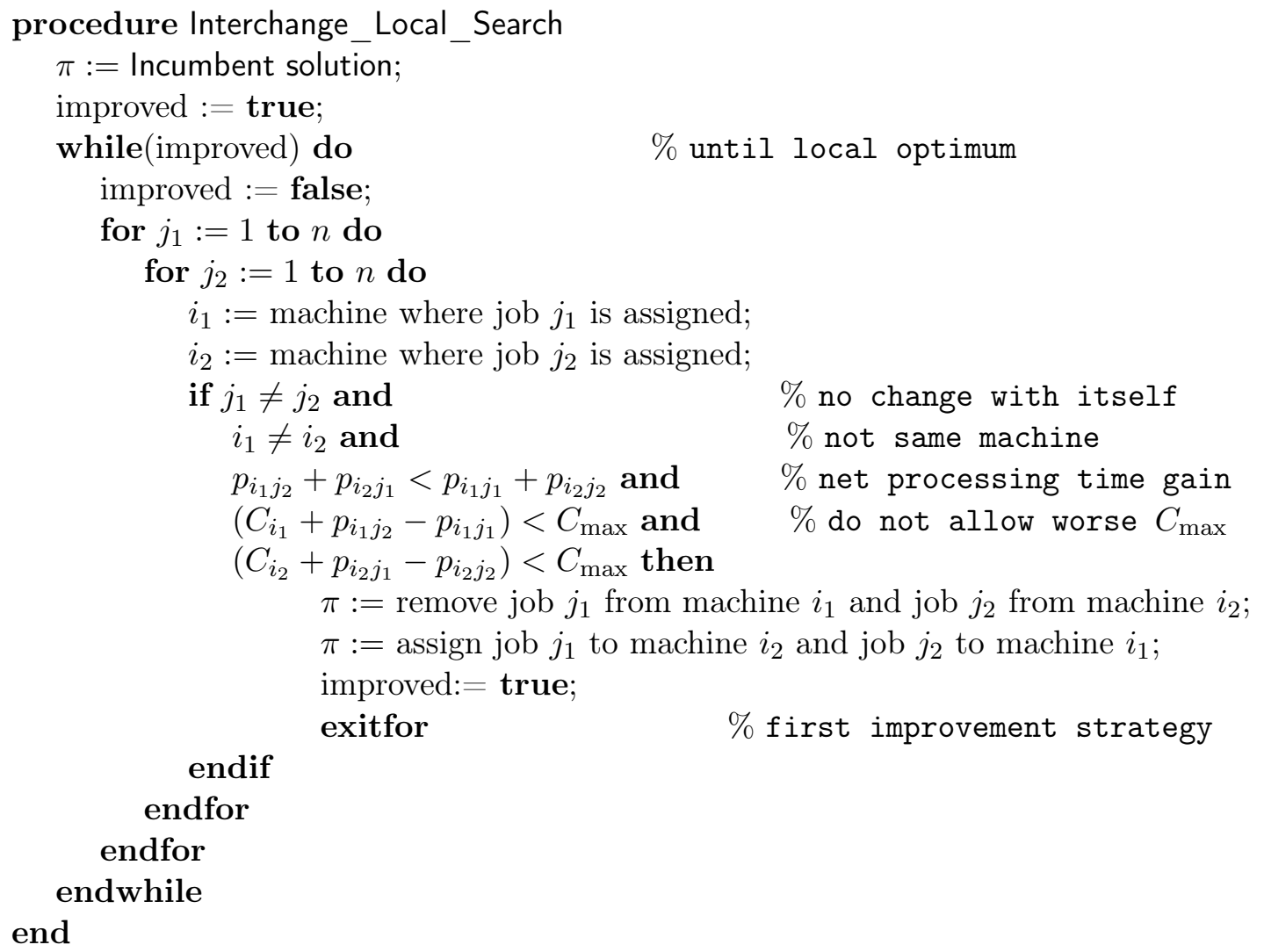

Figure 3: Interchange Local Search procedure employed in the VND phase.

After the VND loop, the solution is a local optimum as regards the insertion and interchange neighborhoods. Therefore, it is not possible to improve the solution any further with additional iterations. At this stage, we apply the solution modification technique depicted in Figure 1 in 
order to scape from this local optimum. We propose two procedures to modify the solution: an Iterated Greedy method (Ruiz and Stützle, 2007) and a Restricted Local Search (RLS) procedure.

\subsection{Iterated Greedy solution modification: IG}

IG methods were introduced in Ruiz and Stützle (2007) for the regular permutation flowshop scheduling problem and later, in Ruiz and Stützle (2008), sequence dependent setup times were additionally considered. Basically, IG starts from a heuristically constructed solution and iterates over two phases: destruction and construction. In the destruction phase, some jobs are randomly extracted from the incumbent solution. Afterwards, these jobs are reinserted one by one in a greedy way, each one in the best position of the partial solution in the construction phase.

We employ this central destruction-reconstruction idea for the solution modification in the proposed methods. More specifically, for the $R / / C_{\max }$ problem, the destruction phase consists in randomly choosing a machine $i$, and, among all jobs assigned to this machine $\left(J_{i}\right)$, one is randomly selected and removed. This procedure is repeated $d$ times. All removed jobs (without repetition) are inserted in a list of removed jobs denoted as $J_{r}$.

In the construction phase all jobs in $J_{r}$ are assigned to the machine $l$ such that $l=\underset{i \in M}{\operatorname{argmin}}\left\{C_{i}+\right.$ $\left.p_{i, J_{r}(k)}\right\}$ where $J_{r}(k)$ denotes the job occupying position $k$ in $J_{r}, k=\{1, \ldots, d\}$.

Note that both the destruction and construction phases are purposedly made as simple as possible. The computational complexity of the application of the IG solution modification procedure is $\mathcal{O}(d m)$. Further details are given in Figure 4,

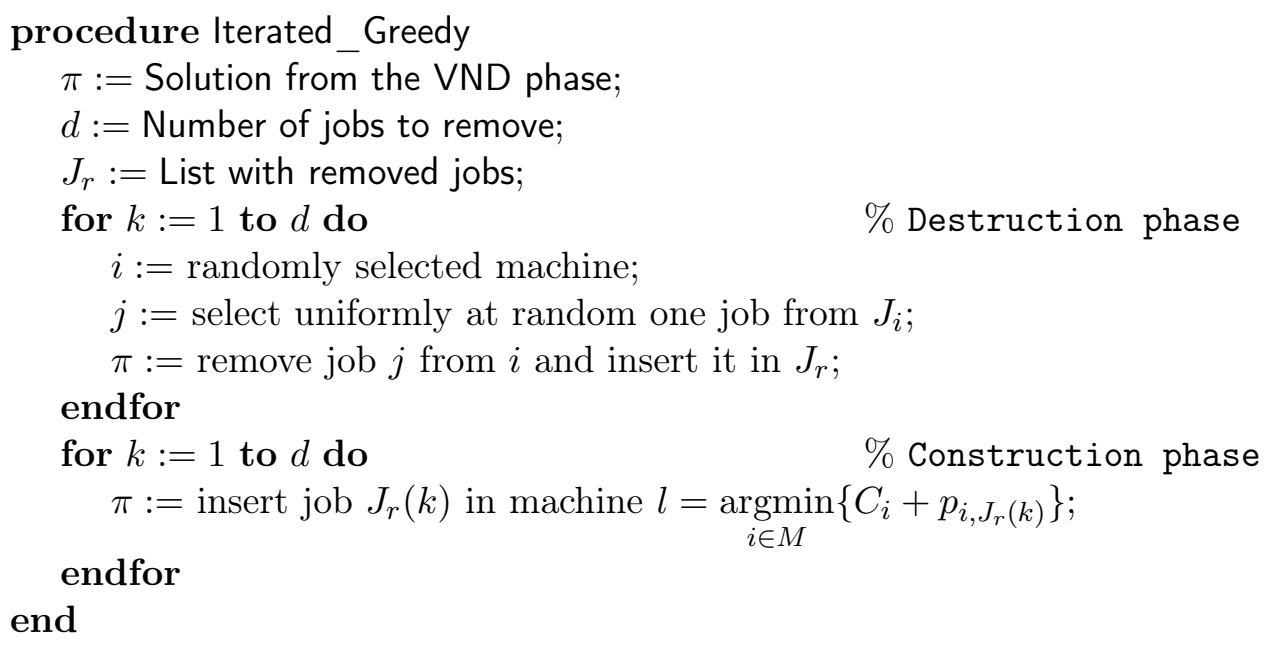

Figure 4: Iterated Greedy (IG) solution modification procedure.

We carried out some short experiments in order to have a first calibration of the $d$ parameter. It is reasonable to bias the removal of jobs towards the machine that is generating the $C_{\max }$, 
i.e., the machine $l=\underset{i \in M}{\operatorname{argmax}}\left\{C_{i}\right\}$ where $C_{\max }=C_{l}$. By doing so, we have greater chances of relocating jobs from $l$ to other machines and of reducing the $C_{\max }$. As a result of these preliminary experiments, we set to remove 20 jobs from this machine $l$ and 20 random jobs uniformly chosen among the remaining machines.

\subsection{Restricted Local Search (RLS) solution modification: NSP and VIR}

Although IG as a solution modification procedure is remarkably simple, other possibly even simpler and faster approaches are also feasible. One possibility is to work over small local searches in restricted neighborhoods. The idea is to relocate a limited number of jobs by applying a different search criteria instead of using the destruction and construction phases like in IG. A fundamental difference we introduce is that in the IG we first apply destruction and an incomplete solution is generated, which is later rebuilt in the construction phase. In the RLS the solution is always complete since we only relocate one job at a time.

We differentiate two RLS methods. The first one is denoted by No Same Place or NSP, and relocates the job to the machine able to finish all its assigned jobs, plus the relocated job, at the earliest possible time. However, we force the job to be moved to a machine different from the original. More specifically, we assign a job $j$, initially assigned to machine $i$, to a machine $l=\underset{h \in M / i}{\operatorname{argmin}}\left\{C_{h}+p_{h j}\right\}$. Note that NSP might actually worsen the $C_{\max }$ value (IG might also deteriorate the solution, but usually, not so strongly). This is indeed, intended. We will comment on the performance of all the proposed methods later.

NSP provides some advantages by moving jobs from their originally assigned machines. However, we observed this to be overly strong and disruptive in some cases. As a result, we propose the second RLS method which is referred to as Virtual or VIR. Contrary to NSP, in VIR we allow a job to return to its original machine. Obviously this has to be done under certain conditions or otherwise no jobs would be relocated (recall that the solution arriving to VIR is already a local optima as regards the insertion and interchange neighborhoods). The condition is that the job $j$ is actually not removed from machine $i$ and then we look for the machine $l=\underset{h \in M}{\operatorname{argmin}}\left\{C_{h}+p_{h j}\right\}$. Therefore, job $j$ could remain in machine $i$ even if assigned twice, i.e., the original job $j$ plus a new "virtual" duplicate. In other words, a given job will stay at its original machine only if that machine is able to process it very effectively. Both NSP and VIR have a computational complexity of $\mathcal{O}(d m)$. Figure 5 explains these two proposed RLS procedures in more detail. 


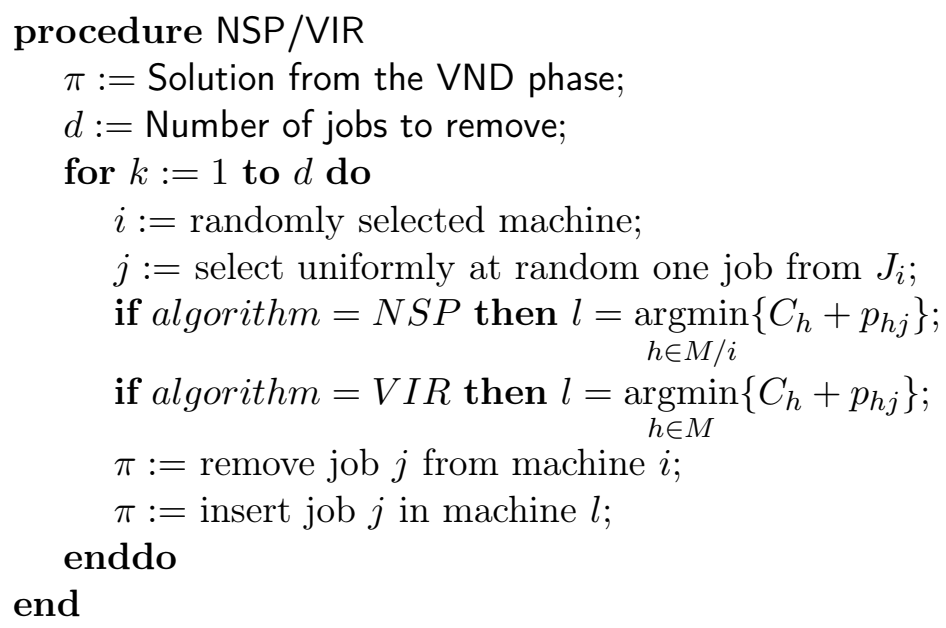

Figure 5: No Same Place (NSP) and Virtual (VIR) Restricted Local Search solution modification procedures.

As it was the case with IG, some preliminary experiments were performed in order to calibrate the parameter $d$ in NSP and VIR. For NSP the best results were obtained by simply removing two jobs, one from the $C_{\max }$ machine and another one from any of the other machines. For VIR, results indicate that five jobs should be removed from the $C_{\max }$ generating machine and five other random jobs from the other machines.

We tried and tested many other algorithm variants and alternatives. These did not produce noticeable gains and/or yielded poor results. For example we tried more elaborated heuristic and a completely random initial solution initialization. Insertion local search was tested with first improvement and interchange with best improvement. We also tried, at some points, the acceptance of solutions with worse $C_{\max }$. We also experimented dynamic values for the $d$ parameter, specially increasing $d$ after a number of unsuccessful iterations of the proposed methods. Most notably, we tested re-initialization operators. We tried a simpler local search stage with no VND loop and only with insertion or interchange local search. We also evaluated the VND loop but with the interchange first and insertion second. While in some cases results improved slightly, we always decided in favor of simplicity.

\subsection{Improving the proposed methods}

The solution modification procedure in the proposed methods, be this by means of IG, NSP or VIR has one clear weak point: at each step, one machine is uniformly selected at random and from that machine, a job assigned to it is also uniformly selected at random. While the advantage of this is approach is simplicity and a certain independence of specific problem knowledge, much 
better solutions can be obtained by making smarter decisions. As a matter of fact, and as has been commented, initial calibrations clearly showed that the machine selection should be biased in favor of the machine generating the $C_{\max }$ value. In the following we explain some techniques that have been applied in order to make the machine and job selection more selective and less random with the aim of improving the results.

\subsubsection{Machine selection}

A bias in the machine selection towards the machine generating the $C_{\max }$ value shows better results. However, there might be more than one machine with $C_{i}=C_{\max }$ and what is more important, a group of machines, while not generating the $C_{\max }$ value, might have $C_{i}$ values very close to the $C_{\max }$.

We studied a series of probability distributions as a function of the different $C_{i}$ values, from the highest $C_{i}\left(C_{\max }\right)$ the lowest $C_{i}$ or $C_{\min }$. We tested many different probability distributions, mainly based in the triangular and inverse triangular numbers. Figure 6 depicts six of the studied distributions.
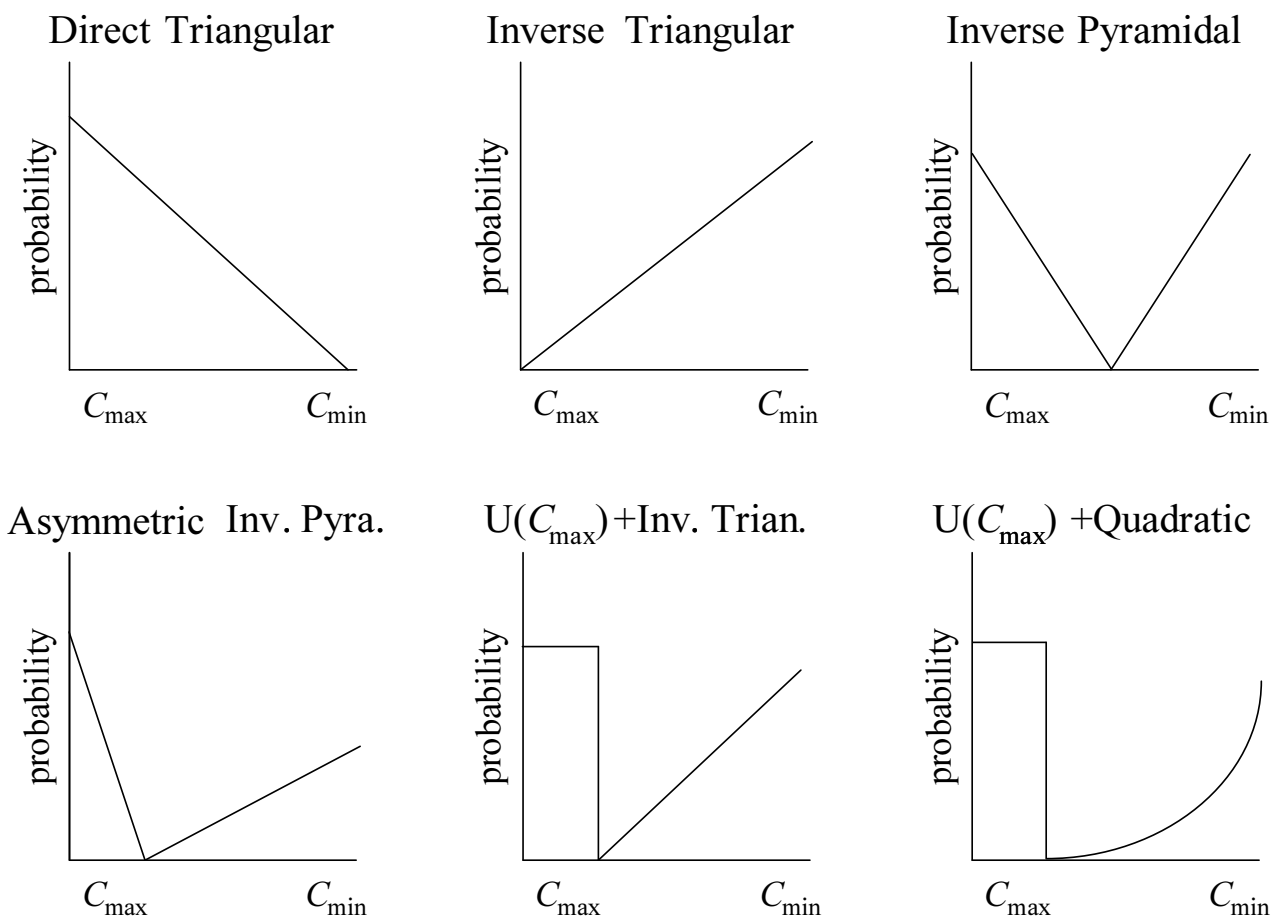

Figure 6: Studied distributions for machine selection.

The Direct Triangular distribution did not improve results in a significant way. However, after testing the Inverse Triangular we obtained much worse results, so it is evident that machines should be selected with greater probability as the $C_{i}$ value increases. However, when we tested the Inverse Pyramidal distribution results improved by a wide margin. The explanation of 
this behavior is interesting. In the $R / / C_{\max }$ problem, and after some iterations of the proposed methods, all machines will have, more or less, the same number of jobs assigned. Furthermore, there would not be large differences between the $C_{\max }$ and $C_{\min }$ values. Under this situation, the insertion neighborhood, and to some extent, the interchange neighborhood are ineffective since there is no machine where to better assign a job as all machines are already "packed". The proposed RLS procedures also tend to get stuck in these cases.

In order to cope with this situation we have to "make room" for jobs from the $C_{\text {max }}$ machines to fit into the machines with lower $C_{i}$ values. The only way to make room is to relocate jobs from machines with low completion times. Therefore, the Inverse Pyramidal distribution, where machines with large and small completion times have greater possibilities of being selected, improves results significantly.

A further refinement comes after considering an Asymmetric Inverse Pyramidal distribution where more bias is given to machines generating higher $C_{i}$ values. Finally, the best result is obtained by uniformly choosing at random any machine generating the $C_{\max }$ value and then an Inverse Triangular distribution as Figure 6 shows. This inverse triangular distribution is easily calculated as follows: we first order all machines with $C_{i}<C_{\max }$ machines in decreasing values of $C_{i}$. The first machine in this list is assigned a value of 1 , the second 3, the third 6 and so on, i.e., the machine in position $h$ has a value which is the sum of the $h$ first integers or $\sum_{i=1}^{h} h=\frac{(h) \cdot(h+1)}{2}$. In order to determine the chosen machine, we randomly generate an integer between 1 and the maximum sum following a uniform distribution. The machine with the closest superior index value to the obtained number is chosen. For example, let us have 5 machines with $C_{i}<C_{\max }$, the indexes are $\{1,3,6,10,15\}$. We draw a uniform integer between 1 and 15 and obtain a 7 . The closest superior index in the list is 10 and therefore the machine in the fourth position of the list is chosen.

\subsubsection{Job selection}

Selecting jobs purely at random is not the wisest approach. Once a machine has been selected, there are many different ways of choosing which job has to be removed from that machine. The best candidates are jobs whose processing times are shorter in other machines as they could be completed earlier if assigned to "faster" machines.

More formally, we have that machine $i$ has been previously selected. Recall that $J_{i}$ represents the set of jobs that have been assigned to this machine and $k$ indicates how many jobs are assigned. Therefore, $J_{i}=\left\{j_{1}, j_{2}, \ldots, j_{k}\right\}$. Then, among the $k$ jobs, we select the job $p$ that has the largest processing time difference with the other machines. In other words, we select the job $p$ according to the following expression:

$$
p=\underset{j_{k} \in J_{i}}{\operatorname{argmax}}\left\{\max _{\substack{l \in M \\ i \neq l}}\left(p_{j_{k}, i}-p_{j_{k}, l}\right)\right\}
$$


There is still one pending issue which is the determination of how many jobs to remove from machines generating $C_{\max }$ and how many from the others. We have to be aware that there are many possible situations. For example, we could have one single machine generating the $C_{\max }$ or we could have an incumbent solution in which all but one machine have $C_{i}$ values equal to $C_{\max }$. As a result of this, we opted for calculating an average of jobs that should be removed from each machine, i.e, if we have 10 machines and $d=20$ then more or less two jobs have to be eliminated from each machine. In order to add bias towards the $C_{\max }$ generating machines, we increase the number of jobs to be removed from these machines by a given percentage over this average of jobs to remove.

\subsubsection{Improved methods}

All previous job and machine selection improvements are included in the proposed methods. Recall that we have the same VND loop and three solution modification techniques: IG, NSP and VIR. The following improved methods are the result of including the selection improvements and some short calibration experiments in order to fix the number of jobs to remove:

- IG+: $d=15$, increase of jobs over the average for machines with $C_{\max }=20 \%$

- $\mathrm{NSP}+: d=5$, increase of jobs over the average for machines with $C_{\max }=20 \%$

- VIR $+: d=10$, increase of jobs over the average for machines with $C_{\max }=40 \%$

As with the regular IG, NSP and VIR variants, we tested and evaluated many other techniques which did not yield good results. We experimented with the slope of the Inverse Triangular distribution or even tried with a Quadratic distribution as Figure 6 shows. We also tried other more elaborated machine and job selection procedures but the additional gains did not justify the added complexity.

\subsection{Algorithm NVST-IG+}

We now propose a complete algorithm that puts together the template of Figure 1 with IG, NSP and VIR. Note that NSP and VIR solution modification procedures are indeed a very special type of local search procedure. Therefore, one idea is to integrate NSP and VIR inside the VND

loop. Considering Hansen and Mladenovic (2001), where neighborhoods should be examined from smallest to largest cardinality, we proceed with this integration as follows:

1. VND loop until local optimum

(a) One single pass of NSP and VIR for all jobs, no worse $C_{\max }$ values

(b) Insertion Local Search, Interchange Local Search. Both until local optimum

2. IG + solution modification procedure 
Therefore we carry out the searches in this order: NSP, VIR, inSertion, inTerchange and IG + . It has to be noted that NSP and VIR can only be applied in a single pass and not until local optima. The reason is clear since after one application of NSP, for example, some jobs are forcibly relocated to other machines. A second application of NSP would return the jobs to their starting assignments. We also have to remark that NSP and VIR inside the VND loop are slightly modified so that worse $C_{\max }$ solutions are not accepted and where all jobs are considered, therefore, as all jobs are selected, there is no need for machine selection.

At this point we would like to emphasize that the resulting NVST-IG + is still a very simple method. Basically we have four neighborhoods that are examined inside a VND loop in a special way and then a simple solution modification procedure based on the Iterated Greedy (IG) methodology is applied. NVST-IG + can be implemented in a very efficient way and all local searches are no more than substractions and additions of processing times with a very low computational complexity per iteration.

As with all other proposed methods, we experimented with many variants of the previous NVST$\mathrm{IG}+$. No relevant improvements could be found.

\section{Computational analysis}

We now test all proposed methods. More specifically, we will carry out comprehensive computational and statistical tests to assess the performance of IG, NSP, VIR, IG+, NSP+, VIR+ and NVST-IG + . We first give a detailed description of the different instance sets that we have employed. After reporting the results, we carry out comprehensive statistical analyses in order to soundly test the significance of the reported results.

\subsection{Benchmark of instances}

In general, there is not a widely available set of benchmark instances for the $R / / C_{\max }$ problem. Authors test their own instances at each paper like for example in Martello et al. (1997), Sourd (2001), Mokotoff and Jimeno (2002) or Ghirardi and Potts (2005). None of these instances are publicly available and their best known solutions are not known.

One instance for the $R / / C_{\max }$ problem is just characterized by $m, n$ and the processing time matrix $p_{i j}$. However, and as we will see, the performance of solution methods is greatly affected by how the processing times in the matrix $p_{i j}$ are distributed. In the literature, the processing times are uniformly distributed in two well known intervals: $U(1,100)$ and $U(10,100)$. Additionally, it is customary in the literature to propose benchmarks where the processing times are job or machine correlated, i.e., short (long) jobs for all machines or slow (fast) machines for all jobs. In the job correlated instances processing times $p_{i j}$ are determined by the expression $p_{i j}=b_{j}+d_{i j}$ where $b_{j}$ and $d_{i j}$ are uniformly distributed values in the ranges $U(1,100)$ and $U(1,20)$, respectively. The processing times in machine correlated instances are governed by a 
similar expression $p_{i j}=a_{i}+c_{i j}$ where $a_{i}$ and $c_{i j}$ are again uniformly distributed in the ranges $U(1,100)$ and $U(1,20)$, respectively.

The previous four distributions for the processing times have not been always employed. For example, the more classical works like that of Glass et al. (1994) or Piersma and van Dijk (1996) primarily used the interval $U(1,100)$, while more recent papers like those of Martello et al. (1997), Mokotoff and Jimeno (2002) or Ghirardi and Potts (2005) have concentrated on the interval $U(10,100)$. The majority of authors include, however, the job and machine correlated instances that were first introduced by Glass et al. (1994).

We propose studying additional sets of instances with different uniform distributions. There are two main reasons for this. First, and as we will show later, IBM-ILOG CPLEX 11.0 solver was able to solve most instances in the interval $U(1,100)$ while only a fraction of the instances could be solved in the interval $U(10,100)$. This leads us to believe that algorithms are far more sensible to the distribution of the processing times as could be initially expected. Second, we believe that distributions $U(1,100)$ and $U(10,100)$ are far from reality. Picture for example the distribution $U(1,100)$ where the differences between the processing times in two machines of the same job can be of up to two orders of magnitude. Therefore, we could have, for a given job $j$ and two machines $i 1$ and $i 2, p_{j, i 1}=1$ and $p_{j, i 1}=100$. If the time units measure hours we have a parallel machine production floor where the same job needs one hour in one machine and 100 hours ( 4 days and 4 hours) in another machine. Such extreme time differences are hardly found in practice. The same reasoning holds for the interval $U(10,100)$, albeit to a lesser extent. As a result, we propose three new unstudied intervals: $U(100,200), U(100,120)$ and $U(1000,1100)$. $U(100,200)$ has the same time units dispersion as $U(1,100)$ but with a lower relative dispersion of processing times for the same job among machines. The interval $U(100,120)$ is proposed as an example with a small dispersion and finally, we propose the interval $U(1000,1100)$ with two objectives: a very small relative dispersion of processing times and also as a test where processing times are extremely large.

We opt for the latest state-of-the-art study of Ghirardi and Potts (2005) for setting the values for $m$ and $n$. More specifically, we test all combinations of $m=\{10,20,30,40,50\}$ and $n=\{100,200,500,1000\}$. Therefore, the largest instances are those of $1000 \times 50$, which is a considerable size indeed. Note that instances smaller than $100 \times 10$ are easily solved to optimality with IBM-ILOG CPLEX 11.0 and are therefore not considered in this paper. In total, there are 20 combinations of $m$ and $n$.

Summing up, we have seven different distributions for the processing times, two standard uniform distributions $(U(1,100)$ and $U(10,100))$, job correlated, machine correlated and three new intervals $(U(100,200), U(100,120)$ and $U(1000,1100))$. For each interval we have 20 com- 
binations of $m$ and $n$ and for each combination we generate 10 instances. Therefore, in total we have $200 \cdot 7=1,400$ instances. We use the instance generator kindly provided by Woclaw (2006). All these instances are available from http://soa.iti.es. The best known solution for each one of the 1,400 instances is obtained by running the MILP model presented in Section 1. with IBM-ILOG CPLEX 11.0 solver with a time limit of two hours on a cluster of $8 \mathrm{PC} / \mathrm{AT}$ computers with Pentium 4 processors running at $3.0 \mathrm{GHz}$ and with $1 \mathrm{~GB}$ of RAM memory and under Windows XP SP3 operating system. The best known solutions are also available from the same web page.

\subsection{Tested algorithms and experimental settings}

As stated in Section 2, the state-of-the-art has been identified as the two phase algorithm of Mokotoff and Jimeno (2002), referred to as Partial and the Recovering Beam Search (RBS) of Ghirardi and Potts (2005). We compare all our proposed methods against these two high performing algorithms and also against IBM-ILOG CPLEX 11.0, which we will simply refer to as CPLEX.

The code for the Partial method of Mokotoff and Jimeno (2002) has been kindly provided by Woclaw (2006). It is implemented in $\mathrm{C}++$ for Linux, where the parameter $r$ has been fixed to 0.5 according to the paper of Mokotoff and Jimeno (2002) and the thesis of Woclaw (2006). Notice that Partial needs a MILP solver and we have employed the same IBM-ILOG CPLEX 11.0 solver under Linux. The code of the RBS from Ghirardi and Potts (2005) has been kindly provided by M. Ghirardi himself. It is coded in $\mathrm{C}++$ for Windows.

Our proposed algorithms IG, NSP, VIR, IG+, NSP + , VIR + and NVST-IG + have been developed with Delphi 2007 and compiled with the optimization flag enabled. Note that all preliminary calibrations that have been briefly commented in Section 3 were carried out just on the interval $U(1,100)$ and for a subset of instances. Moreover, calibrating for each interval would surely result in even a better outcome. However, no further calibrations are carried out to avoid over-fitting deviations in the results.

As regards the CPU time, all our methods as well as CPLEX are stopped at a given elapsed CPU time. We have chosen 15 seconds as a sufficient time as this was the reference time needed by CPLEX to solve all instances of 100 jobs in the interval $U(1,100)$.

Partial, as commented in Section 2 is a deterministic two phase method. The first phase cannot be interrupted. However, the second phase can be stopped at a given time and we also stop this second phase after 15 seconds elapsed CPU time. As a result, Partial CPU times reach for the largest instances more than 60 seconds, which is at least four times more CPU time that our proposed methods. RBS is also a deterministic method but contrary to Partial, it cannot be interrupted. RBS CPU time needs range from very short CPU times of less than 1 second for 100 job instances to more than 100 seconds for 1000 job instances, and this is just for the smallest possible beam width of $w=1$, for $w=3$ the CPU times grow strongly. All algorithms are run in 
the same cluster of 8 computers mentioned earlier. As we can see, results are fully comparable in such an scenario. We have to note though that Delphi 2007 is about a $20 \%$ slower than $\mathrm{C}++$. This together with the fact that our methods are run only for 15 seconds and both Partial and RBS need more CPU time results in a worst case analysis, i.e., our proposed methods are coded in a slower language and run for less CPU time than competing algorithms.

Partial, RBS and CPLEX are deterministic and only one run is necessary. Our seven proposed methods are stochastic and we need to run some replicates in order to better assess the results. We run each one of our seven proposed algorithms five times. The total number of results for the computational experiments is therefore $((7 \cdot 5)+3) \cdot 1,400=53,200$. It has to be noted that this Section entails a comprehensive computational campaign. Counting all instances, algorithms and stopping times (we will see later results with different stopping times) a total of $3.86 \mathrm{CPU}$ years have been employed to obtain all results. The tabulated results for each method will be presented as the relative percentage deviation from the reference solution as follows:

$$
\text { Relative Percentage Deviation }(R P D)=\frac{C_{\max }(i)-C_{\max }^{*}(i)}{C_{\max }^{*}(i)} \cdot 100
$$

Where $C_{\max }^{*}(i)$ is the aforementioned 2 hour CPLEX run (many times optimal or with a very small gap) and $C_{\max }(i)$ is the value obtained by a given algorithm and instance $i$. Before continuing, we present the results of the 2 hour CPLEX run to have a clear reference. Table 1 shows the percentage of instances solved to optimality for CPLEX with a 2 hour time limit. Results are grouped by $m$ and $n$. Each cell represents averages the 10 instances at each group.

\begin{tabular}{|c|c|c|c|c|c|c|c|c|}
\hline$m$ & $n$ & $U(1,100)$ & $U(10,100)$ & $U(100,200)$ & $U(100,120)$ & $U(1000,1100)$ & jobcorre & machcorre \\
\hline \multirow[t]{4}{*}{10} & 100 & 100 & 100 & 100 & 80 & 30 & 0 & 100 \\
\hline & 200 & 100 & 30 & 30 & 60 & 0 & 0 & 40 \\
\hline & 500 & 90 & 40 & 0 & 80 & 0 & 0 & 20 \\
\hline & 1000 & 80 & 20 & 0 & 100 & 0 & 60 & 0 \\
\hline \multirow[t]{4}{*}{20} & 100 & 100 & 50 & 80 & 70 & 40 & 0 & 90 \\
\hline & 200 & 100 & 10 & 10 & 40 & 0 & 0 & 40 \\
\hline & 500 & 70 & 20 & 0 & 30 & 0 & 0 & 10 \\
\hline & 1000 & 50 & 0 & 0 & 30 & 0 & 0 & 0 \\
\hline \multirow[t]{4}{*}{30} & 100 & 100 & 50 & 0 & 50 & 0 & 0 & 90 \\
\hline & 200 & 100 & 0 & 0 & 0 & 0 & 0 & 50 \\
\hline & 500 & 80 & 0 & 0 & 0 & 0 & 0 & 30 \\
\hline & 1000 & 70 & 0 & 0 & 0 & 0 & 0 & 10 \\
\hline \multirow[t]{4}{*}{40} & 100 & 100 & 90 & 10 & 90 & 0 & 0 & 90 \\
\hline & 200 & 100 & 10 & 20 & 100 & 20 & 0 & 70 \\
\hline & 500 & 70 & 0 & 0 & 20 & 0 & 0 & 10 \\
\hline & 1000 & 90 & 0 & 0 & 0 & 0 & 0 & 30 \\
\hline \multirow[t]{4}{*}{50} & 100 & 100 & 90 & 20 & 100 & 100 & 0 & 100 \\
\hline & 200 & 100 & 10 & 10 & 90 & 0 & 0 & 70 \\
\hline & 500 & 80 & 0 & 0 & 40 & 0 & 0 & 50 \\
\hline & 1000 & 80 & 0 & 0 & 0 & 0 & 0 & 50 \\
\hline \multicolumn{2}{|c|}{ Solved instances(\%) } & 88 & 26 & 14 & 49 & 9.5 & 3 & 47.5 \\
\hline
\end{tabular}

Table 1: Percentage of instances solved to optimality by IBM-ILOG CPLEX 11.0 with a time limit of 2 hours. 
As we can see, CPLEX is largely affected by the distribution of the processing times. While the interval $U(1,100)$ is relatively easy, and all instances of 200 jobs or less are solved to optimality, other intervals are much harder. For example, when the processing times are job correlated, CPLEX only manages to solve to optimality a meager $3 \%$ of instances. Notice also how two out of our three new proposed intervals, namely $U(100,200)$ and $U(1000,1100)$ also pose a challenge to CPLEX.

\subsection{Computational results for all intervals}

We present now the full results for all seven intervals. Later we will summarize and will provide some statistical analyses. Tables 2 and 3 show the results for the common intervals $U(1,100)$ and $U(10,100)$. Note that now the acronym "CPLEX" is referred to CPLEX stopped after 15 seconds of CPU time in order to make the results comparable with all other algorithms, including the proposed ones.

\begin{tabular}{cccccccccccc}
\hline$m$ & $n$ & CPLEX & Partial & RBS & IG & NSP & VIR & IG + & NSP + & VIR + & NVST-IG + \\
\hline 10 & 100 & 0.00 & 0.38 & 2.40 & 0.14 & 0.22 & 0.15 & 0.13 & 0.52 & 0.29 & 0.17 \\
& 200 & 0.20 & 0.34 & 1.34 & 0.37 & 0.50 & 0.42 & 0.35 & 0.58 & 0.46 & 0.26 \\
& 500 & 0.29 & 0.31 & 0.97 & 0.57 & 0.57 & 0.53 & 0.45 & 0.53 & 0.49 & 0.37 \\
& 1000 & 0.19 & 0.54 & 0.73 & 0.42 & 0.41 & 0.38 & 0.34 & 0.37 & 0.35 & 0.31 \\
20 & 100 & 0.00 & 0.63 & 2.88 & 0.46 & 1.03 & 1.18 & 0.39 & 1.68 & 1.21 & 0.73 \\
& 200 & 1.75 & 1.39 & 2.28 & 1.68 & 1.82 & 1.68 & 0.94 & 1.94 & 1.51 & 0.98 \\
& 500 & 1.26 & 0.96 & 1.48 & 2.28 & 2.35 & 1.84 & 1.41 & 1.73 & 1.61 & 1.20 \\
& 1000 & 1.35 & 0.91 & 1.09 & 1.44 & 1.46 & 1.29 & 0.87 & 1.01 & 0.98 & 0.83 \\
30 & 100 & 0.00 & 2.99 & 1.21 & 0.63 & 0.85 & 0.86 & 0.63 & 1.07 & 0.74 & 0.00 \\
& 200 & 2.12 & 1.82 & 3.59 & 2.38 & 2.96 & 2.53 & 1.79 & 2.65 & 2.29 & 2.02 \\
& 500 & 3.40 & 2.31 & 2.00 & 4.05 & 4.21 & 2.96 & 2.18 & 2.61 & 2.43 & 1.83 \\
& 1000 & 2.36 & 2.03 & 1.57 & 3.25 & 3.42 & 2.70 & 2.01 & 2.18 & 2.08 & 1.87 \\
40 & 100 & 0.00 & 7.41 & 2.74 & 1.48 & 2.74 & 2.74 & 1.24 & 1.81 & 2.74 & 0.91 \\
& 200 & 3.03 & 1.73 & 4.18 & 3.37 & 4.19 & 3.48 & 2.89 & 3.49 & 3.01 & 2.16 \\
& 500 & 4.15 & 3.64 & 1.82 & 4.19 & 4.86 & 3.52 & 1.97 & 2.33 & 2.23 & 1.46 \\
& 1000 & 4.26 & 4.12 & 2.00 & 4.81 & 5.00 & 3.96 & 2.85 & 2.93 & 2.88 & 2.45 \\
50 & 100 & 0.00 & 7.94 & 0.00 & 1.25 & 1.25 & 1.25 & 1.00 & 1.25 & 1.25 & 0.00 \\
& 200 & 1.60 & 7.31 & 4.87 & 4.71 & 5.19 & 5.32 & 4.55 & 5.17 & 5.14 & 3.69 \\
& 500 & 5.63 & 6.37 & 1.88 & 6.07 & 7.21 & 4.80 & 4.51 & 4.80 & 4.21 & 2.78 \\
& 1000 & 6.05 & 4.50 & 1.57 & 5.66 & 6.21 & 4.38 & 3.17 & 3.44 & 3.13 & 2.70 \\
\hline \multicolumn{2}{l}{ Average } & 1.88 & 2.88 & 2.03 & 2.46 & 2.82 & 2.30 & 1.68 & 2.10 & 1.95 & 1.34 \\
\hline
\end{tabular}

Table 2: Average Relative Percentage Deviations for the interval $U(1,100)$.

For interval $U(1,100)$ we observe a striking result. Partial, which runs for longer time than CPLEX and uses CPLEX in its two phase approach, gives much worse results than CPLEX. In some cases, the differences are very large as for example $100 \times 50$, where CPLEX gives the optimum solution $(0 \% R P D)$ in 15 seconds and Partial gives a $R P D$ of $7.94 \%$. RBS behaves much better than Partial but still lags significantly behind CPLEX. These results support our initial hypothesis that modern versions of CPLEX provide state-of-the-art results, at least for this interval. 
Among our proposed algorithms we have to remark that the simplistic IG, NSP and VIR methods are competitive with Partial and in some cases better than RBS, specially for small instances. Let us recall that for large instances, RBS uses much more CPU time than 15 seconds so our proposed methods are at a clear disadvantage here. In any case, we can safely say that IG, NSP or VIR are much more simpler than RBS or Partial and do not depend on a solver to obtain very competitive solutions in a short amount of time.

In this interval we can also see how the improved versions $\mathrm{IG}+, \mathrm{NSP}+$ and $\mathrm{VIR}+$ significantly improve the results of IG, NSP or VIR. It is clear that a smarter machine and job selection procedures yield significant improvements. Note that the improved versions are run in the same computer and during the same CPU time that the non-improved versions, so the results are fully comparable. We can observe how already VIR + clearly surpasses Partial in most instances and also RBS in most instances, except for the largest ones, $\mathrm{IG}+$ is even better. As a final note for the $U(1,100)$ interval, the NVST-IG + method gives the best overall results, surpassing even CPLEX on most instances.

\begin{tabular}{|c|c|c|c|c|c|c|c|c|c|c|c|}
\hline$m$ & $n$ & CPLEX & Partial & RBS & IG & NSP & VIR & $\mathrm{IG}+$ & $\mathrm{NSP}+$ & VIR+ & NVST-IG+ \\
\hline \multirow[t]{4}{*}{10} & 100 & 0.06 & 0.39 & 1.45 & 0.49 & 0.57 & 0.37 & 0.44 & 0.56 & 0.50 & 0.36 \\
\hline & 200 & 0.25 & 0.27 & 0.80 & 0.52 & 0.43 & 0.30 & 0.27 & 0.36 & 0.28 & 0.21 \\
\hline & 500 & 0.27 & 0.23 & 0.52 & 0.45 & 0.39 & 0.38 & 0.28 & 0.36 & 0.30 & 0.24 \\
\hline & 1000 & 0.19 & 0.73 & 0.39 & 0.26 & 0.23 & 0.22 & 0.18 & 0.19 & 0.19 & 0.16 \\
\hline \multirow[t]{4}{*}{20} & 100 & 0.79 & 0.53 & 2.67 & 0.98 & 1.22 & 0.85 & 0.74 & 1.01 & 0.85 & 0.60 \\
\hline & 200 & 1.66 & 0.90 & 1.52 & 1.67 & 1.15 & 0.94 & 0.71 & 1.01 & 0.87 & 0.57 \\
\hline & 500 & 0.97 & 0.82 & 1.02 & 1.40 & 1.10 & 1.04 & 0.75 & 0.88 & 0.79 & 0.64 \\
\hline & 1000 & 0.83 & 0.66 & 0.47 & 0.84 & 0.75 & 0.71 & 0.53 & 0.62 & 0.58 & 0.51 \\
\hline \multirow[t]{4}{*}{30} & 100 & 2.14 & 1.29 & 4.49 & 1.67 & 1.88 & 1.46 & 0.78 & 1.38 & 0.99 & 0.43 \\
\hline & 200 & 2.96 & 2.40 & 2.16 & 2.44 & 1.80 & 1.82 & 1.25 & 1.55 & 1.48 & 0.68 \\
\hline & 500 & 1.80 & 1.42 & 0.99 & 2.17 & 1.82 & 1.74 & 1.08 & 1.32 & 1.27 & 1.07 \\
\hline & 1000 & 1.38 & 0.98 & 0.64 & 1.44 & 1.25 & 1.24 & 0.87 & 1.01 & 0.96 & 0.98 \\
\hline \multirow[t]{4}{*}{40} & 100 & 0.29 & 1.47 & 4.98 & 2.82 & 3.52 & 2.70 & 2.00 & 2.12 & 2.24 & 0.94 \\
\hline & 200 & 5.02 & 2.05 & 2.68 & 2.93 & 2.46 & 2.58 & 1.23 & 1.98 & 1.48 & 0.82 \\
\hline & 500 & 2.53 & 2.66 & 1.33 & 3.01 & 2.52 & 2.65 & 1.57 & 1.96 & 1.90 & 1.56 \\
\hline & 1000 & 1.75 & 1.72 & 0.81 & 2.00 & 1.71 & 1.77 & 1.27 & 1.42 & 1.37 & 1.38 \\
\hline \multirow[t]{4}{*}{50} & 100 & 0.33 & 0.67 & 6.66 & 0.55 & 1.48 & 0.62 & 0.33 & 0.62 & 0.62 & 0.13 \\
\hline & 200 & 4.36 & 1.80 & 1.80 & 2.43 & 1.91 & 2.27 & 1.08 & 1.52 & 1.48 & 0.16 \\
\hline & 500 & 3.08 & 3.25 & 1.28 & 3.52 & 2.84 & 3.09 & 1.86 & 2.19 & 2.20 & 1.80 \\
\hline & 1000 & 2.05 & 2.05 & 0.83 & 2.48 & 2.18 & 2.23 & 1.62 & 1.83 & 1.79 & 1.81 \\
\hline \multicolumn{2}{|c|}{ Average } & 1.64 & 1.31 & 1.87 & 1.70 & 1.56 & 1.45 & 0.94 & 1.19 & 1.11 & 0.75 \\
\hline
\end{tabular}

Table 3: Average Relative Percentage Deviations for the interval $U(10,100)$.

Moving to the interval $U(10,100)$ we find that Partial gives better average results than CPLEX and RBS. However, we can see how our proposed methods behave relatively better. The nonimproved versions are already very competitive despite their simplicity. The improved versions, specially IG+, are significantly better than the state-of-the-art. Again NVST-IG + yields the best results. In average, NVST-IG + yields results that are $219 \%$ better than CPLEX, 175\% better than Partial and no less than $249 \%$ better than RBS. 
Tables 4 and 5 show the results for instances where processing times are job and machine correlated, respectively. For the first time we see negative average relative percentage deviations in the job correlated instances, specially for RBS, NSP and NVST-IG + . This means that these algorithms, in a very short amount of time, are able to beat the 2-hour reference solution of CPLEX. For the job correlated instances we can see that CPLEX and Partial yields results that are far away from the reference solutions. Again, Partial, using more CPU time, is not even capable of beating CPLEX for 15 seconds CPU time. RBS shows a very good performance and is actually the best method in this benchmark. Our proposed algorithms are much better than CPLEX and Partial and some of them, like NSP+, are competitive with RBS.

\begin{tabular}{|c|c|c|c|c|c|c|c|c|c|c|c|}
\hline$m$ & $n$ & CPLEX & Partial & RBS & IG & NSP & VIR & IG + & $\mathrm{NSP}+$ & VIR+ & NVST-IG+ \\
\hline \multirow[t]{4}{*}{10} & 100 & 0.50 & 0.53 & 0.79 & 0.75 & 0.48 & 0.49 & 0.38 & 0.31 & 0.37 & 0.35 \\
\hline & 200 & 0.29 & 0.26 & 0.35 & 0.49 & 0.36 & 0.42 & 0.24 & 0.22 & 0.25 & 0.22 \\
\hline & 500 & 0.25 & 0.16 & 0.13 & 0.28 & 0.28 & 0.28 & 0.14 & 0.15 & 0.16 & 0.14 \\
\hline & 1000 & 0.15 & 0.09 & 0.09 & 0.11 & 0.13 & 0.13 & 0.08 & 0.09 & 0.09 & 0.09 \\
\hline \multirow[t]{4}{*}{20} & 100 & 1.18 & 2.19 & 1.26 & 1.30 & 0.61 & 1.00 & 0.88 & 0.50 & 0.75 & 0.78 \\
\hline & 200 & 1.64 & 1.18 & 0.56 & 0.63 & 0.38 & 0.50 & 0.37 & 0.33 & 0.41 & 0.37 \\
\hline & 500 & 0.69 & 0.77 & 0.13 & 0.37 & 0.33 & 0.36 & 0.27 & 0.26 & 0.26 & 0.22 \\
\hline & 1000 & 0.70 & 0.47 & 0.05 & 0.27 & 0.25 & 0.27 & 0.22 & 0.22 & 0.23 & 0.20 \\
\hline \multirow[t]{4}{*}{30} & 100 & 2.32 & 4.73 & 0.98 & 1.82 & 0.50 & 1.04 & 0.95 & 0.44 & 1.01 & 0.71 \\
\hline & 200 & 2.82 & 2.50 & 0.57 & 1.26 & 0.61 & 0.96 & 0.80 & 0.65 & 0.80 & 0.64 \\
\hline & 500 & 2.21 & 1.16 & 0.07 & 0.44 & 0.27 & 0.38 & 0.29 & 0.25 & 0.31 & 0.17 \\
\hline & 1000 & 1.56 & 1.25 & -0.02 & 0.21 & 0.16 & 0.18 & 0.16 & 0.14 & 0.16 & 0.13 \\
\hline \multirow[t]{4}{*}{40} & 100 & 2.42 & 7.21 & 1.22 & 4.03 & 1.93 & 2.03 & 2.41 & 1.39 & 2.54 & 2.24 \\
\hline & 200 & 3.12 & 3.49 & -0.24 & 1.34 & 0.11 & 0.79 & 0.62 & 0.36 & 0.69 & 0.33 \\
\hline & 500 & 3.95 & 1.75 & -0.06 & 0.35 & 0.04 & 0.25 & 0.14 & 0.10 & 0.16 & 0.02 \\
\hline & 1000 & 2.59 & 1.77 & -0.11 & 0.12 & 0.03 & 0.08 & 0.05 & 0.03 & 0.05 & 0.01 \\
\hline \multirow[t]{4}{*}{50} & 100 & 3.18 & 7.83 & 2.54 & 5.06 & 4.02 & 3.32 & 2.34 & 1.60 & 2.92 & 2.17 \\
\hline & 200 & 4.97 & 5.09 & -0.65 & 2.28 & 0.54 & 1.42 & 1.27 & 0.91 & 1.27 & 0.94 \\
\hline & 500 & 4.83 & 3.34 & -0.42 & 0.41 & -0.10 & 0.18 & 0.06 & 0.02 & 0.10 & -0.10 \\
\hline & 1000 & 4.57 & 2.87 & -0.28 & 0.14 & -0.02 & 0.08 & 0.05 & 0.01 & 0.06 & -0.02 \\
\hline \multicolumn{2}{|c|}{ Average } & 2.20 & 2.43 & 0.35 & 1.08 & 0.54 & 0.71 & 0.59 & 0.40 & 0.63 & 0.48 \\
\hline
\end{tabular}

Table 4: Average Relative Percentage Deviations for the interval where jobs have correlated processing times.

For machine correlated instances we observe how Partial significantly improves results (and beats CPLEX) and at the same time RBS gives the worst results from the comparison, and by a significant margin. We want to emphasize these results here as RBS was the best method for job correlated instances. An important conclusion that can be drawn is that Partial and RBS are not particularly robust as regards the distribution of the processing time. For machine correlated instances we can see how all our propose methods, starting with IG and ending with NVST-IG+ are significantly better than CPLEX, Partial or RBS. 


\begin{tabular}{|c|c|c|c|c|c|c|c|c|c|c|c|}
\hline$m$ & $n$ & CPLEX & Partial & RBS & $\mathrm{IG}$ & NSP & VIR & $\mathrm{IG}+$ & $\mathrm{NSP}+$ & $\mathrm{VIR}+$ & NVST-IG+ \\
\hline \multirow[t]{4}{*}{10} & 100 & 0.00 & 0.10 & 1.65 & 0.28 & 0.27 & 0.14 & 0.24 & 0.23 & 0.21 & 0.25 \\
\hline & 200 & 0.45 & 0.37 & 1.47 & 0.56 & 0.42 & 0.47 & 0.52 & 0.49 & 0.49 & 0.53 \\
\hline & 500 & 0.28 & 0.15 & 0.73 & 0.60 & 0.55 & 0.54 & 0.69 & 0.64 & 0.64 & 0.65 \\
\hline & 1000 & 0.27 & 0.20 & 0.34 & 0.64 & 0.60 & 0.59 & 0.61 & 0.63 & 0.61 & 0.59 \\
\hline \multirow[t]{4}{*}{20} & 100 & 0.08 & 0.07 & 3.44 & 0.51 & 0.37 & 0.39 & 0.44 & 0.37 & 0.37 & 0.43 \\
\hline & 200 & 0.91 & 0.53 & 1.16 & 0.42 & 0.28 & 0.32 & 0.33 & 0.31 & 0.31 & 0.29 \\
\hline & 500 & 0.86 & 0.56 & 1.65 & 0.72 & 0.66 & 0.67 & 0.71 & 0.71 & 0.69 & 0.72 \\
\hline & 1000 & 0.48 & 0.39 & 0.51 & 0.87 & 0.84 & 0.85 & 0.88 & 0.88 & 0.85 & 0.88 \\
\hline \multirow[t]{4}{*}{30} & 100 & 0.00 & 0.08 & 3.77 & 0.44 & 0.37 & 0.30 & 0.40 & 0.32 & 0.32 & 0.25 \\
\hline & 200 & 1.28 & 1.15 & 2.27 & 0.76 & 0.53 & 0.58 & 0.55 & 0.48 & 0.44 & 0.50 \\
\hline & 500 & 1.09 & 1.17 & 0.82 & 0.79 & 0.69 & 0.71 & 0.75 & 0.72 & 0.69 & 0.68 \\
\hline & 1000 & 0.96 & 1.19 & 1.20 & 1.02 & 1.03 & 1.02 & 0.97 & 1.00 & 1.00 & 0.98 \\
\hline \multirow[t]{4}{*}{40} & 100 & 0.11 & 0.00 & 4.18 & 0.22 & 0.23 & 0.15 & 0.20 & 0.15 & 0.20 & 0.15 \\
\hline & 200 & 1.58 & 0.96 & 4.37 & 0.67 & 0.50 & 0.52 & 0.55 & 0.42 & 0.46 & 0.49 \\
\hline & 500 & 1.67 & 1.86 & 2.53 & 0.76 & 0.68 & 0.69 & 0.69 & 0.70 & 0.67 & 0.64 \\
\hline & 1000 & 2.52 & 2.28 & 1.78 & 0.75 & 0.74 & 0.74 & 0.73 & 0.74 & 0.71 & 0.69 \\
\hline \multirow[t]{4}{*}{50} & 100 & 0.29 & 0.00 & 5.37 & 0.47 & 0.53 & 0.35 & 0.41 & 0.29 & 0.35 & 0.32 \\
\hline & 200 & 1.91 & 0.69 & 4.29 & 0.82 & 0.56 & 0.65 & 0.56 & 0.51 & 0.50 & 0.33 \\
\hline & 500 & 2.72 & 2.87 & 2.99 & 1.00 & 0.97 & 0.94 & 0.81 & 0.81 & 0.83 & 0.77 \\
\hline & 1000 & 5.19 & 4.23 & 2.75 & 0.93 & 0.96 & 0.95 & 0.85 & 0.89 & 0.85 & 0.82 \\
\hline \multicolumn{2}{|c|}{ Average } & 1.13 & 0.94 & 2.36 & 0.66 & 0.59 & 0.58 & 0.60 & 0.56 & 0.56 & 0.55 \\
\hline
\end{tabular}

Table 5: Average Relative Percentage Deviations for the interval where machines have correlated processing times.

Finally, we show the results of the three new intervals $U(100,200), U(100,120)$ and $U(1000,1100)$ in Tables 6, 7 and 8 .

\begin{tabular}{|c|c|c|c|c|c|c|c|c|c|c|c|}
\hline$m$ & $n$ & CPLEX & Partial & RBS & IG & NSP & VIR & IG + & $\mathrm{NSP}+$ & $\mathrm{VIR}+$ & NVST-IG+ \\
\hline \multirow[t]{4}{*}{10} & 100 & 0.07 & 0.09 & 1.22 & 0.33 & 0.30 & 0.19 & 0.26 & 0.29 & 0.24 & 0.26 \\
\hline & 200 & 0.69 & 0.43 & 1.06 & 0.40 & 0.23 & 0.29 & 0.33 & 0.38 & 0.35 & 0.31 \\
\hline & 500 & 0.29 & 0.23 & 0.49 & 0.26 & 0.13 & 0.18 & 0.21 & 0.23 & 0.22 & 0.21 \\
\hline & 1000 & 0.21 & 0.31 & 0.22 & 0.13 & 0.08 & 0.11 & 0.12 & 0.11 & 0.13 & 0.11 \\
\hline \multirow[t]{4}{*}{20} & 100 & 0.38 & 0.47 & 1.07 & 0.39 & 0.37 & 0.32 & 0.32 & 0.43 & 0.35 & 0.30 \\
\hline & 200 & 0.50 & 0.83 & 1.42 & 0.74 & 0.47 & 0.64 & 0.50 & 0.56 & 0.59 & 0.49 \\
\hline & 500 & 1.05 & 0.66 & 1.04 & 0.75 & 0.58 & 0.67 & 0.71 & 0.76 & 0.77 & 0.69 \\
\hline & 1000 & 0.80 & 0.67 & 0.64 & 0.50 & 0.38 & 0.46 & 0.46 & 0.50 & 0.49 & 0.40 \\
\hline \multirow[t]{4}{*}{30} & 100 & 1.90 & 2.81 & 1.01 & 0.21 & 0.16 & 0.03 & -0.01 & 0.02 & -0.01 & -0.10 \\
\hline & 200 & 1.15 & 2.73 & 0.01 & 0.11 & -0.29 & -0.12 & -0.22 & -0.22 & -0.20 & -0.30 \\
\hline & 500 & 0.91 & 1.47 & 0.57 & 0.78 & 0.56 & 0.66 & 0.59 & 0.57 & 0.59 & 0.55 \\
\hline & 1000 & 0.84 & 0.93 & 0.56 & 0.66 & 0.59 & 0.64 & 0.58 & 0.59 & 0.61 & 0.46 \\
\hline \multirow[t]{4}{*}{40} & 100 & 1.38 & 2.43 & 0.99 & 0.47 & 0.42 & 0.34 & 0.22 & 0.16 & 0.18 & 0.07 \\
\hline & 200 & 1.01 & 1.06 & 0.89 & 0.83 & 0.48 & 0.83 & 0.43 & 0.48 & 0.66 & 0.42 \\
\hline & 500 & 0.48 & 2.15 & 0.32 & 0.54 & 0.35 & 0.43 & 0.31 & 0.30 & 0.33 & 0.28 \\
\hline & 1000 & 0.98 & 1.18 & 0.70 & 0.69 & 0.58 & 0.66 & 0.57 & 0.63 & 0.61 & 0.58 \\
\hline \multirow[t]{4}{*}{50} & 100 & 0.28 & 0.14 & 1.66 & 0.14 & 0.22 & 0.35 & 0.10 & 0.09 & 0.36 & 0.11 \\
\hline & 200 & 0.85 & 0.68 & 1.12 & 0.95 & 0.55 & 0.96 & 0.54 & 0.58 & 0.85 & 0.52 \\
\hline & 500 & 0.69 & 0.98 & 0.69 & 0.84 & 0.58 & 0.80 & 0.51 & 0.59 & 0.64 & 0.51 \\
\hline & 1000 & 0.71 & 0.69 & 0.54 & 0.60 & 0.48 & 0.58 & 0.49 & 0.51 & 0.54 & 0.47 \\
\hline \multicolumn{2}{|c|}{ Average } & 0.76 & 1.05 & 0.81 & 0.52 & 0.36 & 0.45 & 0.35 & 0.38 & 0.42 & 0.32 \\
\hline
\end{tabular}

Table 6: Average Relative Percentage Deviations for the interval $U(100,200)$.

For the interval $U(100,200)$ we can see how all our proposed methods are, on average, significantly better than CPLEX, Partial or RBS. We want to emphasize that this is a worst case 
comparison as both Partial and RBS use more CPU time than 15 seconds and are coded in $\mathrm{C}++$, which is more efficient when compared to Delphi. Therefore, we can safely say that our proposed methods produce better results even in unfavorable circumstances.

\begin{tabular}{|c|c|c|c|c|c|c|c|c|c|c|c|}
\hline$m$ & $n$ & CPLEX & Partial & RBS & IG & NSP & VIR & IG + & $\mathrm{NSP}+$ & VIR + & NVST-IG+ \\
\hline \multirow[t]{4}{*}{10} & 100 & 0.06 & 0.19 & 0.24 & 0.10 & 0.06 & 0.06 & 0.07 & 0.09 & 0.09 & 0.08 \\
\hline & 200 & 0.10 & 0.13 & 0.19 & 0.08 & 0.04 & 0.05 & 0.06 & 0.06 & 0.07 & 0.06 \\
\hline & 500 & 0.15 & 0.29 & 0.23 & 0.07 & 0.04 & 0.06 & 0.08 & 0.10 & 0.09 & 0.09 \\
\hline & 1000 & 0.16 & 0.28 & 0.13 & 0.05 & 0.03 & 0.04 & 0.07 & 0.07 & 0.07 & 0.07 \\
\hline \multirow[t]{4}{*}{20} & 100 & 0.20 & 0.28 & 0.22 & 0.11 & 0.06 & 0.10 & 0.06 & 0.06 & 0.06 & 0.05 \\
\hline & 200 & 0.24 & 0.26 & 0.22 & 0.15 & 0.08 & 0.13 & 0.08 & 0.09 & 0.11 & 0.09 \\
\hline & 500 & 0.16 & 0.23 & 0.18 & 0.10 & 0.07 & 0.09 & 0.07 & 0.08 & 0.09 & 0.07 \\
\hline & 1000 & 0.25 & 0.26 & 0.16 & 0.08 & 0.06 & 0.08 & 0.08 & 0.09 & 0.09 & 0.08 \\
\hline \multirow[t]{4}{*}{30} & 100 & 0.15 & 0.35 & 0.18 & 0.15 & 0.04 & 0.09 & 0.00 & 0.00 & 0.01 & 0.00 \\
\hline & 200 & 0.44 & 0.68 & 0.04 & 0.10 & 0.03 & 0.07 & 0.03 & 0.02 & 0.03 & 0.01 \\
\hline & 500 & 0.39 & 0.42 & 0.11 & 0.17 & 0.13 & 0.15 & 0.12 & 0.11 & 0.11 & 0.10 \\
\hline & 1000 & 0.12 & 0.14 & -0.05 & 0.07 & 0.05 & 0.07 & 0.05 & 0.05 & 0.05 & 0.03 \\
\hline \multirow[t]{4}{*}{40} & 100 & 0.03 & 0.40 & 0.10 & 0.15 & 0.00 & 0.04 & 0.00 & 0.00 & 0.00 & 0.00 \\
\hline & 200 & 0.12 & 0.18 & 0.06 & 0.08 & 0.06 & 0.08 & 0.03 & 0.04 & 0.07 & 0.03 \\
\hline & 500 & 0.07 & 0.86 & -0.02 & 0.06 & 0.01 & 0.05 & -0.02 & -0.01 & -0.02 & -0.02 \\
\hline & 1000 & 0.16 & 0.14 & 0.10 & 0.07 & 0.06 & 0.06 & 0.05 & 0.06 & 0.05 & 0.05 \\
\hline \multirow[t]{4}{*}{50} & 100 & 0.05 & 0.94 & 0.30 & 0.12 & 0.03 & 0.14 & 0.01 & 0.04 & 0.09 & 0.00 \\
\hline & 200 & 0.05 & 0.32 & 0.07 & 0.06 & 0.02 & 0.08 & 0.02 & 0.02 & 0.02 & 0.01 \\
\hline & 500 & 0.11 & 0.10 & 0.04 & 0.09 & 0.05 & 0.08 & 0.01 & 0.03 & 0.03 & 0.01 \\
\hline & 1000 & 0.07 & 0.10 & 0.03 & 0.03 & 0.02 & 0.03 & 0.01 & 0.02 & 0.01 & 0.01 \\
\hline \multicolumn{2}{|c|}{ Average } & 0.15 & 0.33 & 0.13 & 0.09 & 0.05 & 0.08 & 0.04 & 0.05 & 0.06 & 0.04 \\
\hline
\end{tabular}

Table 7: Average Relative Percentage Deviations for the interval $U(100,120)$.

\begin{tabular}{cccccccccccc}
\hline$m$ & $n$ & CPLEX & Partial & RBS & IG & NSP & VIR & IG + & NSP + & VIR + & NVST-IG + \\
\hline 10 & 100 & 0.06 & 0.04 & 0.14 & 0.04 & 0.03 & 0.02 & 0.03 & 0.04 & 0.03 & 0.03 \\
& 200 & 0.04 & 0.06 & 0.13 & 0.05 & 0.02 & 0.03 & 0.04 & 0.05 & 0.04 & 0.04 \\
& 500 & 0.06 & 0.03 & 0.09 & 0.03 & 0.02 & 0.02 & 0.03 & 0.04 & 0.04 & 0.03 \\
& 1000 & 0.08 & 0.08 & 0.07 & 0.02 & 0.02 & 0.02 & 0.03 & 0.04 & 0.03 & 0.03 \\
20 & 100 & 0.04 & 0.07 & 0.18 & 0.03 & 0.03 & 0.02 & 0.01 & 0.03 & 0.03 & 0.02 \\
& 200 & 0.09 & 0.08 & 0.17 & 0.07 & 0.04 & 0.06 & 0.04 & 0.06 & 0.06 & 0.05 \\
& 500 & 0.10 & 0.04 & 0.09 & 0.05 & 0.03 & 0.04 & 0.04 & 0.04 & 0.04 & 0.03 \\
& 1000 & 0.10 & 0.07 & 0.08 & 0.04 & 0.03 & 0.04 & 0.05 & 0.05 & 0.05 & 0.05 \\
30 & 100 & 0.17 & 0.36 & 0.23 & 0.02 & 0.01 & -0.01 & -0.01 & -0.01 & -0.01 & -0.02 \\
& 200 & 0.09 & 0.72 & -0.03 & -0.07 & -0.11 & -0.09 & -0.10 & -0.10 & -0.10 & -0.11 \\
& 500 & 0.02 & 0.94 & 0.03 & 0.04 & 0.01 & 0.03 & 0.02 & 0.02 & 0.02 & 0.01 \\
& 1000 & 0.10 & 0.07 & 0.07 & 0.07 & 0.06 & 0.06 & 0.06 & 0.06 & 0.06 & 0.05 \\
40 & 100 & 0.13 & 0.35 & 0.33 & 0.04 & 0.03 & 0.02 & 0.01 & 0.01 & 0.01 & 0.00 \\
& 200 & 0.10 & 0.09 & 0.29 & 0.05 & 0.02 & 0.05 & 0.02 & 0.04 & 0.04 & 0.02 \\
& 500 & 0.03 & 1.19 & 0.03 & 0.04 & 0.02 & 0.03 & 0.02 & 0.02 & 0.02 & 0.01 \\
& 1000 & 0.08 & 0.08 & 0.09 & 0.05 & 0.04 & 0.05 & 0.04 & 0.04 & 0.04 & 0.04 \\
50 & 100 & 0.01 & 0.01 & 0.77 & 0.01 & 0.01 & 0.01 & 0.00 & 0.00 & 0.02 & 0.00 \\
& 200 & 0.07 & 0.11 & 0.52 & 0.06 & 0.03 & 0.07 & 0.03 & 0.04 & 0.05 & 0.03 \\
& 500 & 0.08 & 0.09 & 0.23 & 0.07 & 0.05 & 0.07 & 0.04 & 0.05 & 0.06 & 0.04 \\
& 1000 & 0.06 & 0.06 & 0.13 & 0.05 & 0.04 & 0.05 & 0.04 & 0.04 & 0.05 & 0.04 \\
\hline \multicolumn{1}{l}{ Average } & 0.08 & 0.23 & 0.18 & 0.04 & 0.02 & 0.03 & 0.02 & 0.03 & 0.03 & 0.02 \\
\hline
\end{tabular}

Table 8: Average Relative Percentage Deviations for the interval $U(1000,1100)$.

Interval $U(100,120)$ is not particularly hard for all methods as the shown relative percentage 
deviations are very close to zero. In this benchmark, CPLEX under two hours was capable of solving a large number of instances and the observed gap in those that could not be solved was very small. Still, once again, all our proposed methods are significantly better than the competition. The best methods are IG + and NVST-IG + with average results of $0.04 \%$. The best method from the competition is RBS, with a $0.13 \%$. In other words, our best proposed algorithms are at least $325 \%$ better than the competition. Lastly, for interval $U(1000,1100)$ we have a similar situation where IG + and NVST-IG + are the best with $0.02 \%$ and the best competitor is CPLEX with $0.08 \%$, i.e., $400 \%$ worse. We would like to point out that in these three new benchmarks, CPLEX is significantly better than Partial and RBS is most cases.

In order to have a clear overall picture, Table 9 shows all seven tested benchmarks along with all proposed methods, CPLEX, Partial and RBS. The table just gathers all previously presented results.

\begin{tabular}{ccccccccccc}
\hline Interval & CPLEX & Partial & RBS & IG & NSP & VIR & IG + & NSP + & VIR + & NVST-IG + \\
\hline$U(1,100)$ & 1.88 & 2.88 & 2.03 & 2.46 & 2.82 & 2.3 & 1.68 & 2.10 & 1.95 & $\mathbf{1 . 3 4}$ \\
$U(10,100)$ & 1.64 & 1.31 & 1.87 & 1.7 & 1.56 & 1.45 & 0.94 & 1.19 & 1.11 & $\mathbf{0 . 7 5}$ \\
$U(100,200)$ & 0.76 & 1.05 & 0.81 & 0.52 & 0.36 & 0.45 & 0.35 & 0.38 & 0.42 & $\mathbf{0 . 3 2}$ \\
$U(100,120)$ & 0.15 & 0.33 & 0.13 & 0.09 & 0.05 & 0.08 & $\mathbf{0 . 0 4}$ & 0.05 & 0.06 & $\mathbf{0 . 0 4}$ \\
$U(1000,1100)$ & 0.08 & 0.23 & 0.18 & 0.04 & $\mathbf{0 . 0 2}$ & 0.03 & $\mathbf{0 . 0 2}$ & 0.03 & 0.03 & $\mathbf{0 . 0 2}$ \\
jobcorre & 2.20 & 2.43 & $\mathbf{0 . 3 5}$ & 1.08 & 0.54 & 0.71 & 0.59 & 0.40 & 0.63 & 0.48 \\
machcorre & 1.13 & 0.94 & 2.36 & 0.66 & 0.59 & 0.58 & 0.60 & 0.56 & 0.56 & $\mathbf{0 . 5 5}$ \\
\hline Average & 1.12 & 1.31 & 1.10 & 0.94 & 0.85 & 0.80 & 0.60 & 0.67 & 0.68 & $\mathbf{0 . 5 0}$ \\
\hline A. typical & 1.71 & 1.89 & 1.65 & 1.48 & 1.38 & 1.26 & 0.95 & 1.07 & 1.06 & $\mathbf{0 . 7 8}$ \\
\hline A.typical= Average of more typical intervals: $U(1,100) ;$ & $U(10,100) ;$ Jobs Correlated; Machines Correlated
\end{tabular}

Table 9: Summary results for all benchmarks and algorithms. Bold (italics) figures represent best (worst) results, respectively.

We have calculated two global averages. The first one is the average results for each method of all seven tested benchmarks, i.e., an average relative percentage deviation from the 2-hour CPLEX reference solution for all 1,400 instances. The second global average contains the four "typical" intervals, i.e.: $U(1,100), U(10,100)$, Jobs Correlated, Machines Correlated. We show the two averages in order to avoid a bias in the final results possibly due to our three new proposed benchmarks. Looking at these averages we can see how all our seven proposed algorithms yield better results than the competition in our computational settings. At this point, we have to remark that our proposed methods are remarkably simple and make no use of commercial solvers. Among all tested benchmarks, RBS manages to beat NVST-IG + for job correlated instances. However, RBS uses, on average, around 25 seconds of CPU time and NVST-IG + has been run for just 15 seconds. We will later show some additional results for longer CPU times.

So far we have just shown average results. We need to carry out some statistical testing in order to guarantee that the observed differences in the average results are indeed statistically 
significant. We carry out a Design of Experiments (DOE, Montgomery, 2009) where we study a single factor, i.e., the type of algorithm at 10 levels and the average relative percentage deviation is the response variable. We take all previous results where each instance is considered a treatment and there are 1,400 results for CPLEX, Partial and RBS and 7,000 results (five replicates) for each one of our seven proposed methods. Recall that in total there are 53,200 results. The results of the DOE are analyzed by means of the single factor Analysis of Variance (ANOVA) technique. We check the three main hypotheses of the parametric ANOVA: normality, homocedasticity and independence of the residuals. With such a large set of results, the residuals from the ANOVA easily satisfied all three hypotheses. Figure 7 shows the means plot with Tukey HSD intervals with 95\% confidence level. Recall that overlapping intervals indicates that no statistically significant difference exists among the overlapped means.

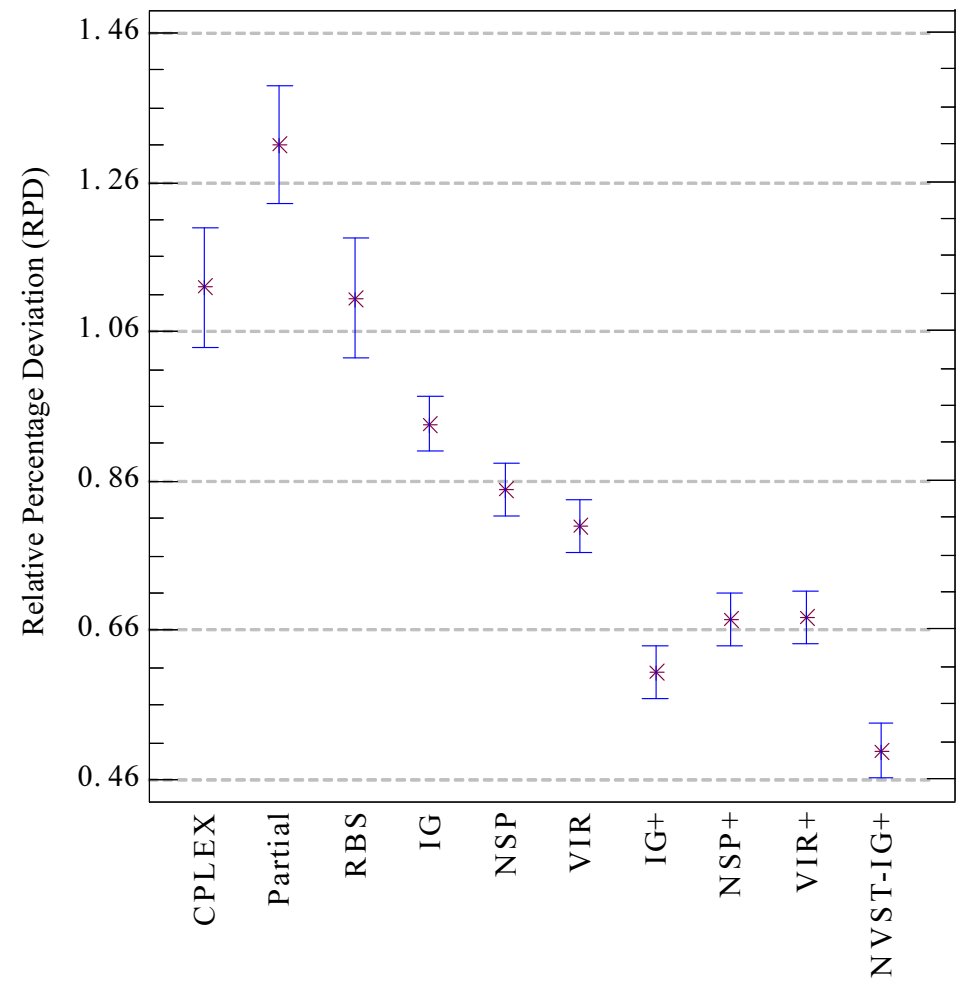

Figure 7: Means plot and Tukey HSD intervals with 95\% confidence level for all tested algorithms and all instances.

As can be seen, Partial is indeed statistically worse than CPLEX. Of course, this applies to the overall average. Notice that the Tukey intervals are fairly wide, which means that different results could be observed by zooming in for each different benchmark. RBS and CPLEX are statistically equivalent. All our seven proposed methods are statistically better than CPLEX, Partial and RBS. However, NSP and VIR are equivalent. The same can be said about the improved NSP + and VIR + versions. Finally, NVST-IG + is statistically better than all other tested methods. 
All previous results have been carried out with all our seven proposed methods and CPLEX stopping at 15 seconds CPU time. Recall that Partial is stopped after $15 \mathrm{CPU}$ seconds have elapsed in the second phase, and therefore the total time is larger. Recall also that the CPU time of RBS is not controllable and therefore it has been run for an approximate overall average of 25 seconds. A final question remains. Are the good results of NVST-IG + maintained with lower or higher CPU time? We aim now at answering this question. We have carried out additional experiments where the stopping CPU time has been set at 5, 25, 300,600, 1,800, 3,600 and 7,200 seconds. Again, the exact times can only be controlled for CPLEX and our seven proposed methods. These times are used for the second phase of Partial and only up to 300 seconds. RBS has been tested at 25 seconds (more or less its natural stopping time, on average) and at 300 seconds after increasing the beam width. All these results are shown in Table 10.

\begin{tabular}{rrrccccccc}
\hline \multirow{2}{*}{ Time } & Algorithms & $U(1,100)$ & $U(10,100)$ & $U(100,200)$ & $U(100,120)$ & $U(1000,1100)$ & Jobcorre & Machcorre & Average \\
\hline 5 & Partial & 3.93 & 2.25 & 1.22 & 0.36 & 0.27 & 2.89 & 1.49 & 1.77 \\
& CPLEX & 3.45 & 2.53 & 0.98 & 0.20 & 0.09 & 3.92 & 2.70 & 1.98 \\
& NVST-IG + & $\mathbf{1 . 7 8}$ & $\mathbf{1 . 0 6}$ & $\mathbf{0 . 4 1}$ & $\mathbf{0 . 0 6}$ & $\mathbf{0 . 0 3}$ & $\mathbf{0 . 7 1}$ & $\mathbf{0 . 6 6}$ & $\mathbf{0 . 6 7}$ \\
\hline \multirow{2}{*}{25} & Partial & 2.45 & 1.22 & 0.69 & 0.28 & 0.18 & 2.12 & 0.73 & 1.10 \\
& CPLEX & 1.41 & 1.42 & 0.58 & 0.12 & 0.04 & 1.75 & 0.69 & 0.86 \\
& NVST-IG+ & $\mathbf{1 . 2 0}$ & $\mathbf{0 . 6 1}$ & $\mathbf{0 . 2 7}$ & $\mathbf{0 . 0 4}$ & $\mathbf{0 . 0 2}$ & 0.38 & $\mathbf{0 . 5 1}$ & $\mathbf{0 . 4 3}$ \\
& RBS & 2.03 & 1.87 & 0.81 & 0.13 & 0.18 & $\mathbf{0 . 3 5}$ & 2.36 & 1.10 \\
\hline \multirow{2}{*}{300} & Partial & 1.59 & 0.33 & 0.20 & 0.21 & 0.09 & 1.23 & $\mathbf{0 . 1 0}$ & 0.54 \\
& CPLEX & $\mathbf{0 . 3 5}$ & 0.29 & 0.24 & 0.04 & 0.02 & 0.45 & 0.12 & 0.22 \\
& NVST-IG+ & 0.67 & $\mathbf{0 . 1 9}$ & $\mathbf{0 . 1 4}$ & $\mathbf{0 . 0 2}$ & $\mathbf{0 . 0 0}$ & 0.05 & 0.38 & $\mathbf{0 . 2 1}$ \\
& RBS & 0.87 & 0.85 & 0.39 & 0.07 & 0.08 & $\mathbf{- 0 . 2 0}$ & 1.17 & 0.46 \\
\hline \multirow{2}{*}{600} & CPLEX & $\mathbf{0 . 2 4}$ & 0.20 & 0.17 & 0.03 & 0.01 & 0.27 & $\mathbf{0 . 0 7}$ & $\mathbf{0 . 1 4}$ \\
& NVST-IG + & 0.58 & $\mathbf{0 . 0 9}$ & $\mathbf{0 . 1 0}$ & $\mathbf{0 . 0 1}$ & $\mathbf{0 . 0 0}$ & $\mathbf{- 0 . 0 2}$ & 0.35 & 0.16 \\
\hline \multirow{2}{*}{1,800} & CPLEX & $\mathbf{0 . 1 2}$ & 0.11 & 0.08 & 0.02 & 0.01 & 0.12 & $\mathbf{0 . 0 3}$ & $\mathbf{0 . 0 7}$ \\
& NVST-IG+ & 0.53 & $\mathbf{0 . 0 2}$ & $\mathbf{0 . 0 7}$ & $\mathbf{0 . 0 1}$ & $\mathbf{- 0 . 0 1}$ & $\mathbf{- 0 . 1 1}$ & 0.32 & 0.12 \\
\hline \multirow{2}{*}{3,600} & CPLEX & $\mathbf{0 . 0 5}$ & 0.06 & $\mathbf{0 . 0 2}$ & 0.01 & 0.00 & 0.07 & $\mathbf{0 . 0 1}$ & $\mathbf{0 . 0 3}$ \\
& NVST-IG + & 0.49 & $\mathbf{- 0 . 0 3}$ & 0.05 & $\mathbf{0 . 0 0}$ & $\mathbf{- 0 . 0 1}$ & $\mathbf{- 0 . 2}$ & 0.29 & 0.09 \\
\hline \multirow{2}{*}{7,200} & CPLEX & $\mathbf{0 . 0 0}$ & 0.00 & $\mathbf{0 . 0 0}$ & $\mathbf{0 . 0 0}$ & 0.00 & 0.00 & $\mathbf{0 . 0 0}$ & $\mathbf{0 . 0 0}$ \\
& NVST-IG+ & 0.38 & $\mathbf{- 0 . 0 7}$ & 0.03 & $\mathbf{0 . 0 0}$ & $\mathbf{- 0 . 0 1}$ & $\mathbf{- 0 . 2 6}$ & 0.27 & 0.05 \\
\hline
\end{tabular}

Table 10: Average results for the best methods and different CPU stopping criteria. Bold (italics) figures represent best (worst) results, respectively.

One striking result is that NVST-IG + is the best method when run for an extremely short amount of time ( 5 seconds) and the total average relative percentage deviation from the 2-hour CPLEX solution is $0.67 \%$. This is a $296 \%$ better than CPLEX and $264 \%$ better than Partial. We think that these results are noteworthy since in just a mere 5 seconds, very good average results can be obtained without using a solver. For 25 and 300 seconds, NVST-IG + is still the best method, on average. RBS improves significantly when compared to the 25 seconds stopping 
time but does not manage to beat CPLEX or NVST-IG + . Partial is the worst method in all tested cases (CPU time $\leq 300)$. As we can see, although all previous computational evaluations were carried out with a 15 seconds CPU time limit, similar conclusions can be drawn with less and more CPU time.

For longer processing times of 10, 30, 60 and 120 minutes, we have just compared the two best methods, i.e., CPLEX and NVST-IG+. Overall, with more time, CPLEX gets obviously better. After all, IBM-ILOG CPLEX 11.0. applies state-of-the-art Branch and Cut exact algorithms and it is expected to beat any other method under extended CPU time. However, it is quite remarkable how NVST-IG + does not stall and steadily improves results across all instances. Actually, for the interval $U(1000,1100)$ and where processing times are job correlated, NVST$\mathrm{IG}+$ improves the results of the 2-hour CPLEX already for 10 minutes, which is quite remarkable.

As a matter of fact, we evaluate CPLEX vs. NVST-IG + for even more different stopping points. We carry out a two-factor ANOVA whose means interaction plot is shown in Figure 8 , Again, it must be noted that the means plot shows overall averages. We can observe that for up to 60 seconds, it is, on average, better to use NVST-IG + instead of CPLEX. From that point and all the way up to two hours, it is statistically equivalent to employ CPLEX or NVST$\mathrm{IG}+$. However, we can see how the curve of CPLEX falls below that of NVST-IG + around 600 seconds. Therefore, for some specific benchmarks and instances, CPLEX is expected to provide better results.

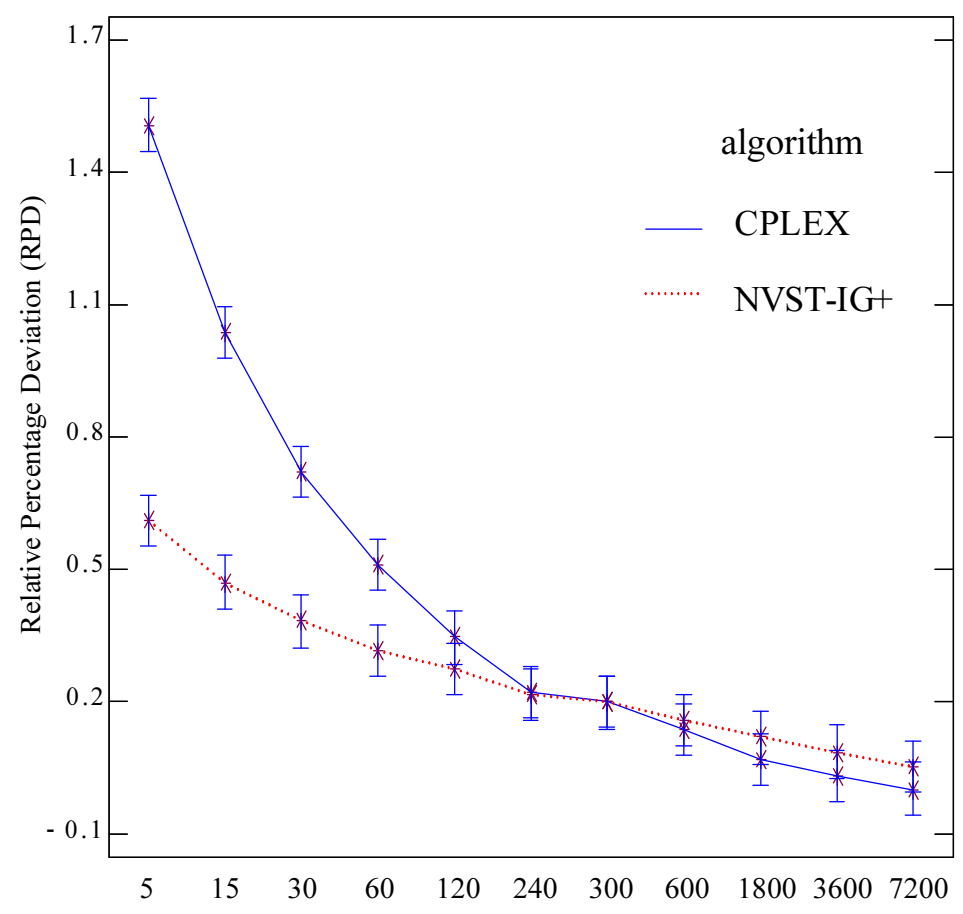

Figure 8: Means interaction plot and Tukey HSD intervals with 95\% confidence level for CPLEX and NVST-IG + and all instances. 


\section{Conclusions and future research}

In this paper we have proposed seven new algorithms for the unrelated parallel machine scheduling problem under makespan criterion or $R / / C_{\max }$. The methods presented are remarkable simple and are mainly composed of a very simple solution initialization, a Variable Neighborhood Descent loop (VND, Mladenovic and Hansen, 1997; Hansen and Mladenovic, 2001), and a solution modification procedure. Three basic algorithms: IG, NSP and VIR have been initially presented. Then we have improved these methods by selecting jobs and machines in a more smart way, creating the improved $\mathrm{IG}+, \mathrm{NSP}+$ and VIR + methods. Later, all the ideas have been joined in a still remarkable simple NVST-IG + method.

A comprehensive benchmark test of 1,400 instances has been employed in order to compare all presented algorithms against state-of-the-art methods, identified as IBM-ILOG CPLEX 11.0., Partial of Mokotoff and Jimeno (2002) and RBS of Ghirardi and Potts (2005). An exhaustive computational campaign has been carried out which has needed almost 4 years of CPU time. All results have been statistically tested. In most situations, our presented algorithms have yielded results that are statistically better, and by a significant margin, that the aforementioned stateof-the-art procedures. We think that these results are remarkable specially if we consider the inherent simplicity of the local search-based proposed methods. Other interesting results is that recent versions of CPLEX are actually competitive, improving the results of Partial and RBS in most situations.

Future research stems from the consideration of more elaborated neighborhood definitions inside the VND loop, together with a further improvement in the selection of jobs and machines which could further bolster results. We are also interested in applying the proposed techniques to other more sophisticated parallel machines problems, like those resulting from the addition of sequence dependent setup times and/or to other objectives, like those based on job's due dates.

\section{Acknowledgments}

The authors are partly funded by the Spanish Department of Science and Innovation (research projects ref. DPI2008-03511/DPI and IAP-020100-2008-11).

The authors would like to sincerely thank A. Woclaw and M. Ghirardi for their help and for providing the valuable codes that have been employed in the computational evaluation of the Partial and RBS methods. 


\section{References}

Cheng, T. C. E. and Sin, C. C. S. (1990). A state-of-the-art review of parallel-machine scheduling research. European Journal of Operational Research, 47(3):271-292.

Davis, E. and Jaffe, J. M. (1981). Algorithms for scheduling tasks on unrelated processors. Journal of the ACM, 28(4):721-736.

De, P. and Morton, T. E. (1980). Scheduling to minimise makespan on unequal parallel processors. Management Science, 11(4):586-602.

de la Croce, F., Ghirardi, M., and Tadei, R. (2004). Recovering beam search: Enhancing the beam search approach for combinatorial optimization problems. Journal of Heuristics, 10(1):89-104.

Dillenberger, C., Escudero, L. F., Wollensak, A., and Wu, Z. (1994). On practical resourceallocation for production planning and scheduling with period overlapping setups. European Journal of Operational Research, 75(2):275-286.

Gairing, M., Monien, B., and Woclaw, A. (2007). A faster combinatorial approximation algorithm for scheduling unrelated parallel machines. Theoretical Computer Science, 380(1-2):87-99.

Garey, M. R. and Johnson, D. S. (1979). Computers and intractability: A guide to the theory of NP-completeness. Freeman. San Francisco.

Ghirardi, M. and Potts, C. N. (2005). Makespan minimization for scheduling unrelated parallel machines: A recovering beam search approach. European Journal of Operational Research, 165(2):457-467.

Glass, C. A., Potts, C. N., and Shade, P. (1994). Unrelated parallel machine scheduling using local search. Mathematical and Computer Modelling, 20(2):41-52.

Graham, R. L. (1969). Bounds on multiprocessing timing anomalies. SIAM Journal on Applied Mathematics, 17(2):416-429.

Graham, R. L., Lawler, E. L., Lenstra, J. K., and Rinnooy Kan, A. H. G. (1979). Optimization and approximation in deterministic sequencing and scheduling: A survey. Annals of Discrete Mathematics, 5:287-326.

Hansen, P. and Mladenovic, N. (2001). Variable neighborhood search: Principles and applications. European Journal of Operational Research, 130(3):449-467.

Hariri, A. M. A. and Potts, C. N. (1991). Heuristics for scheduling unrelated parallel machines. Computers $\&$ Operations Research, 18(3):323-331.

Horowitz, E. and Sahni, S. (1976). Exact and approximate algorithms for scheduling nonidentical processors. Journal of the ACM, 23(2):317-327.

Ibarra, O. H. and Kim, C. E. (1977). Heuristic algorithms for scheduling independent tasks on nonidentical processors. Journal of the ACM, 24(2):280-289.

Lenstra, J. K., Rinnooy Kan, A. H. G., and Brucker, P. (1977). Complexity of machine scheduling problems. Annals of Discrete Mathematics, 1.

Lenstra, J. K., Shmoys, D. B., and Tardos, E. (1990). Approximation algorithms for scheduling unrelated parallel machines. Mathematical Programming, 46(3):259-271.

Mansini, R. and Speranza, M. G. (1999). Heuristic algorithms for the portfolio selection problem with minimum transaction lots. European Journal of Operational Research, 114(2):219-233.

Martello, S., Soumis, F., and Toth, P. (1997). Exact and approximation algorithms for makespan minimization on unrelated parallel machines. Discrete Applied Mathematics, 75(2):169-188.

McNaughton, R. (1959). Scheduling with deadlines and loss functions. Management Science, $6(1): 1-12$.

Mladenovic, N. and Hansen, P. (1997). Variable neighborhood search. Computers \& Operations Research, 24(11):1097-1100. 
Mokotoff, E. (2001). Parallel machine scheduling problems: A survey. Asia-Pacific Journal of Operational Research, 18(2):193-242.

Mokotoff, E. and Chretienne, P. (2002). A cutting plane algorithm for the unrelated parallel machine scheduling problem. European Journal of Operational Research, 141(3):515-525.

Mokotoff, E. and Jimeno, J. L. (2002). Heuristics based on partial enumeration for the unrelated parallel processor scheduling problem. Annals of Operations Research, 117(1-4):133-150.

Montgomery, D. C. (2009). Design and Analysis of Experiments. Wiley, New York, seventh edition.

Ow, P. S. and Morton, T. E. (1988). Filtered beam search in scheduling. International Journal of Production Research, 26(1):35-62.

Piersma, N. and van Dijk, W. (1996). A local search heuristic for unrelated parallel machine scheduling with efficient neighborhood search. Mathematical and Computer Modelling, 24(9):11-19.

Pinedo, M. L. (2005). Planning and Scheduling in Manufacturing and Services. Springer Series in Operations Research. Springer, New York, USA.

Pinedo, M. L. (2008). Scheduling: Theory, Algorithms, and Systems. Springer, New York, USA, third edition.

Potts, C. N. (1985). Analysis of a linear-programming heuristic for scheduling unrelated parallel machines. Discrete Applied Mathematics, 10(2):155-164.

Ruiz, R. and Stützle, T. (2007). A simple and effective iterated greedy algorithm for the permutation flowshop scheduling problem. European Journal of Operational Research, 177(3):20332049 .

Ruiz, R. and Stützle, T. (2008). An iterated greedy heuristic for the sequence dependent setup times flowshop problem with makespan and weighted tardiness objectives. European Journal of Operational Research, 187(3):1143-1159.

Shchepin, E. V. and Vakhania, N. (2005). An optimal rounding gives a better approximation for scheduling unrelated machines. Operations Research Letters, 33(2):127-133.

Shmoys, D. B. and Tardos, E. (1993). An approximation algorithm for the generalized assignment problem. Mathematical Programming, 62(3):461-474.

Sourd, F. (2001). Scheduling tasks on unrelated machines: Large neighborhood improvement procedures. Journal of Heuristics, 7(6):519-531.

Srivastava, B. (1998). An effective heuristic for minimising makespan on unrelated parallel machines. Journal of the Operational Research Society, 49(8):886-894.

Sule, D. R. (2008). Production Planning and Industrial Scheduling: Examples, Case Studies and Applications. CRC Press, Boca Raton, USA, second edition.

van de Velde, S. L. (1993). Duality based algorithms for scheduling unrelated parallel machines. ORSA Journal on Computing, 5(5):192-205.

Woclaw, A. (2006). Scheduling Unrelated Parallel Machines. Algorithms, Complexity, and Performance. PhD thesis, Fakultat fur Elektrotechnik, Informatik und Mathematik der Universitat Paderborn, Deutschland. 